\title{
O)
}

\section{Dynamic Mass of a Reaction Wheel Including Gyroscopic Effects: an Experimental Approach}

\begin{tabular}{|c|c|}
\hline Journal: & AIAA Journal \\
\hline Manuscript ID & 2016-06-J055398 \\
\hline Manuscript Type: & Full Paper \\
\hline Date Submitted by the Author: & 01-Jun-2016 \\
\hline Complete List of Authors: & $\begin{array}{l}\text { Addari, Daniele; University of Surrey, Surrey Space Centre } \\
\text { Aglietti, Guglielmo; University of Surrey, } \\
\text { Remedia, Marcello; University of Surrey, Electronic Engineering }\end{array}$ \\
\hline Subject Index Category: & $\begin{array}{l}03500 \text { Vibration < } 00000 \text { AIRCRAFT TECHNOLOGY, CONVENTIONAL, } \\
\text { STOL/VTOL, } 80800 \text { Structural Dynamics and Characterization < } 80000 \\
\text { STRUCTURAL MECHANICS AND MATERIALS, } 80200 \text { Dynamic Model } \\
\text { Analysis < } 80000 \text { STRUCTURAL MECHANICS AND MATERIALS }\end{array}$ \\
\hline $\begin{array}{r}\text { Select ONE Subject Index for } \\
\text { the Table of } \\
\text { Contents. <br>This is where } \\
\text { your paper will show up in the } \\
\text { Table of Contents: }\end{array}$ & 80000 STRUCTURAL MECHANICS AND MATERIALS \\
\hline
\end{tabular}




\title{
Gyroscopic Effects: an Experimental Approach
}

\author{
Daniele Addari ${ }^{\mathrm{a}}$ and Guglielmo S. Aglietti ${ }^{\mathrm{b}}$ and Marcello Remedia ${ }^{\mathrm{c}}$ \\ University of Surrey, Guildford, Surrey, United Kingdom, GU2 7XH
}

\begin{abstract}
In the recent years, driven by the increasingly stringent stability requirements imposed by some satellites' payloads (e.g. the new generation of optical instruments), the issue of accurate on-board spacecraft microvibration modelling has attracted significant interest from engineers and scientists. This paper investigates the microvibrationinduced phenomenon on a cantilever configured reaction wheel assembly including sub and higher harmonic amplifications due to modal resonances and broadband noise. A mathematical model of the reaction wheel assembly is developed and validated against experimental test results. The model is capable to represent each configuration in which the reaction wheel assembly will operate whether it is hard-mounted on a dynamometric platform or suspended free-free. The outcomes of this analysis are used to establish a novel methodology to retrieve the dynamic mass of the reaction wheel assembly in its operative range of speeds. An alternative measurement procedure has been developed for this purpose, showing to produce good estimates over a wide range of frequency using a less complex test campaign compared to typical dynamic mass setups. Furthermore, the gyroscopic effect influence in the reaction wheel assembly response is thoroughly examined both analytically and experimentally. Finally, to what extent the noise affects the convergence of the novel approach is investigated.
\end{abstract}

\footnotetext{
a Ph.D. student, Surrey Space Centre, d.addari@surrey.ac.uk, AIAA Student Member.

b Professor, Surrey Space Centre, g.aglietti@surrey.ac.uk.

c Research fellow, Surrey Space Centre, m.remedia@surrey.ac.uk.
} 


\section{Nomenclature}

$A \quad=\quad$ harmonic amplitude coefficient

$\mathbf{a}=$ power spectral density matrix of accelerations

$\mathbf{C} \quad=\quad$ damping matrix

$c \quad=\quad$ generalized damping coefficient

$\mathbf{D} \quad=\quad$ dynamic mass matrix

$D \quad=\quad$ generalized dynamic mass coefficient

$d \quad=\quad$ distance between system centre of mass and flywheel centre of mass

$f \quad=\quad$ force vector

$\mathbf{G} \quad=\quad$ gyroscopic matrix

$H \quad=\quad$ harmonic fraction

$h \quad=\quad$ distance between system centre of mass and wheel-base centre of mass

$I R=$ second moment of inertia

$I Z=$ moment of inertia with respect to spin axis

$\mathbf{K}=$ stiffness matrix

$k \quad=\quad$ generalized stiffness coefficient

$l=$ height distance from mass imbalance and flywheel centre of mass

$\mathbf{M}=$ mass matrix

$M \quad=\quad$ generalized mass coefficient

$m \quad=\quad$ flywheel mass imbalance

$\boldsymbol{q} \quad=\quad$ generalized vector of coordinates

$R \quad=\quad$ cage radius

$r \quad=\quad$ radial distance between flywheel mass imbalance and flywheel centre of mass

$\mathbf{T}=$ transformation matrix

$T \quad=\quad$ kinetic energy

$t \quad=\quad$ generalized time unit

$U \quad=\quad$ potential energy

$v \quad=\quad$ distance between wheel-base and ground

$W \quad=\quad$ broadband $/$ random noise 


\section{Sub/Superscripts}

$\mathrm{b}=$ wheel-base

c $\quad=\quad$ coupled configuration

fis $\quad=\quad$ free-free boundary condition

$\mathrm{H}=$ hermitian

$\mathrm{hm}=$ hard-mounted boundary condition

$\mathrm{i} \quad=$ generalized index

$\mathrm{m}=$ flywheel mass imbalance

$\mathrm{mp} \quad=\quad$ mounting point

$\mathrm{r}=$ radial rotational mode

$\mathrm{s}=$ system

$\mathrm{t}=$ radial translational mode

$\mathrm{w} \quad=\quad$ flywheel

$\mathrm{xz}=\mathrm{xz}$-plane

$\mathrm{yz}=\mathrm{yz}$-plane

$\mathrm{z}=$ axial translational mode 


\section{Introduction}

Some modern spacecraft demand stringent pointing requirements and platform stability which pose a significant challenge for the control and mitigation of microvibrations on-board spacecraft. The James Webb Space Telescope [1], the Solar Observatory Solar-C [2] but also the low-cost end market (e.g. SSTL300-S1 platform [3, 4] and Skybox [5]), all carry vibration sensitive instruments on-board (e.g. high resolution cameras aiming to achieve $1 \mathrm{~m}$ ground resolution), thereby, even displacements in the order of micro-meters of the instrument mounting interface may significantly affect the light of sight of the instrument, dramatically reducing its performance. In this context we refer to microvibration as mechanical vibrations with amplitudes in the range of micro-g's $(\mu g)$ acting in a range of frequency from a few $\mathrm{Hz}$ up to $1 \mathrm{kHz}[6]$. These disturbances are typically induced by the on-board satellite equipment, in particular fast rotating mechanisms such as wheel assemblies [7], cryo-coolers, and antenna and solar pointing mechanisms [8]. Other possible sources of microvibration are thrusters and switches [9]. Among the various sources, however, Reaction Wheel Assemblies (RWAs) are generally regarded as the largest and most important ones [10, 11]. The induced disturbances are transmitted through the satellite structure towards the payloads or sensitive instruments, impacting on their correct functioning. Moreover, due to coupled dynamics between the source and the supporting structure [12], estimates of the microvibration effects are even more challenging [13]. Therefore, the modeling of microvibration sources and their prediction have drawn considerable interest throughout the years and an extensive amount of work can be found in literature, i.e. [7, 14-19]. Moreover, their mitigation and control using passive or active damping systems have been thoroughly investigated, for example [16, 17, 20-22], with the final objective to estimate and minimise payload pointing errors [23, 24].

Currently, the most common approach to evaluate the microvibrations induced by a RWA is to carry out experiments grounding (i.e. hard-mounted boundary condition) the RWA on a dynamometric table (e.g. Kistler table) and measure the forces and moments whilst the RWA is in operation $[14,15,17,25-27]$. The data are subsequently used as input to the satellite Finite Element (FE) model to predict the induced effects on the payload. The concept of this methodology is, however, flawed because grounded RWA microvibrations measurements are not representative 
of the real environment in which the RWA will operate as, in practice, it works in a zero "g" environment and is mounted on a flexible structure (i.e. the satellite). In fact, when a RWA is installed on a flexible supporting structure, its disturbances excite the modes of the structure which, in turn, perturb the RWA itself, thus creating coupled dynamics [12]. Hence the actual loads at the interface are different from those obtained in a hard-mounted boundary condition. For this reason, in order to accurately reproduce the dynamics when a RWA is mounted on its supporting structure, the apparent masses (also referred as dynamic masses or their inverse, the accelerances) of the source and the supporting structure at the interface mounting point between the two bodies have to be considered [15]. In [28], a method to analyse the RWA-structure coupled microvibrations was presented, considering both the RWA and the supporting structure as rigid bodies. The dynamic mass concept and importance, together with its dependence on the frequency were also introduced. The work was subsequently extended in [14, 29-31], where a practical method to retrieve the RWA dynamic mass was developed. Despite the outcomes provided better results than those obtained from the hard-mounted boundary condition, the RWA dynamic mass was measured with the flywheel not spinning (in this study referred as zero-speed condition). In contrast, when the flywheel is in operation (non-zero speed condition), it produces a dual effect: microvibration disturbances and variations of the RWA structural response as the speed increases. The latter is due to the gyroscopic effect which significantly influences the dynamic mass of the RWA. Its parameters will, in fact, not only change as a function of the frequency but also of the speed of the rotary flywheel. In [29, 32], analytical expressions of RWA dynamic mass including the gyroscopic effect based on a RWA hard-mounted microvibration model were derived and used for coupled microvibration analysis. Although the results were improved, the modes of vibration of the RWA were not included hence limiting the quality of predictions. These analyses were expanded in [33], where an experimental method to measure the driving point dynamic mass including the gyroscopic effect was discussed and validated. This involved a RWA-seismic mass coupled system suspended free-free using elastic cords and mini-shakers used to apply unit forces and moments at the interface mounting point location in one Degree of Freedom (DoF) at the time. The data were then used to estimate the coupled loads exchanged at the interface between the two bodies, including the internal 
vibration modes of the RWA. The results showed some improvement with respect to previous works. However, the test configuration is quite challenging as several items have to be suspended by means of elastic cords; moreover, it is also important that the mini-shakers are perfectly aligned with the DoF they have to excite to not generate coupled responses, making this methodology less likely to be applicable.

In this article, an alternative and new approach to retrieve the dynamic mass of a RWA including the gyroscopic effect is presented. It involves measurements of the forces and moments derived from a hard-mounted boundary condition and the accelerations at the RWA mounting interface when the RWA is suspended free-free. The test setup is significantly simpler than that used in [33]. This involves the use of two dedicated platforms and common force sensors and accelerometers. A RWA model presented in [34] is used for this purpose. Characteristics of the RWA in hardmounted and free-free boundary conditions are discussed in this article and both experimental and analytical results are used for dynamic mass studies. The dynamic mass of a RWA including the gyroscopic effect is initially obtained from its FE model and subsequently derived processing the experimental data. Coupled microvibration theory is also briefly introduced. Finally, discussions of the microvibration measurement method presented in this work are provided with emphasis given to the influence of the background noise on the convergence of the novel methodology.

\section{RWA Analytical Model}

In this study, a cantilever configured RWA supported by a semi-rigid suspension system is considered. The RWA disturbance model was developed in [17] and subsequently re-elaborated in [35]. An energy approach is here implemented to derive the generalized Equation of Motion (EoM) through Lagrange's equation [36]. Under linear assumptions, analytical expressions of kinetic energy, potential energy and work done can be expressed for the mass balanced flywheel case and subsequently for the mass imbalanced case. Finally, the linearized EoM of a fully mass imbalanced RWA are derived for both hard-mounted and free-free suspended boundary conditions. To clarify the terminology adopted in this article, suspension refers to the link between the flywheel and the rest of the mechanism; subscripts $w$ and $b$ refer to the flywheel and wheel-base, respectively. The model is subsequently implemented to predict the dynamic mass of the RWA in both static and 


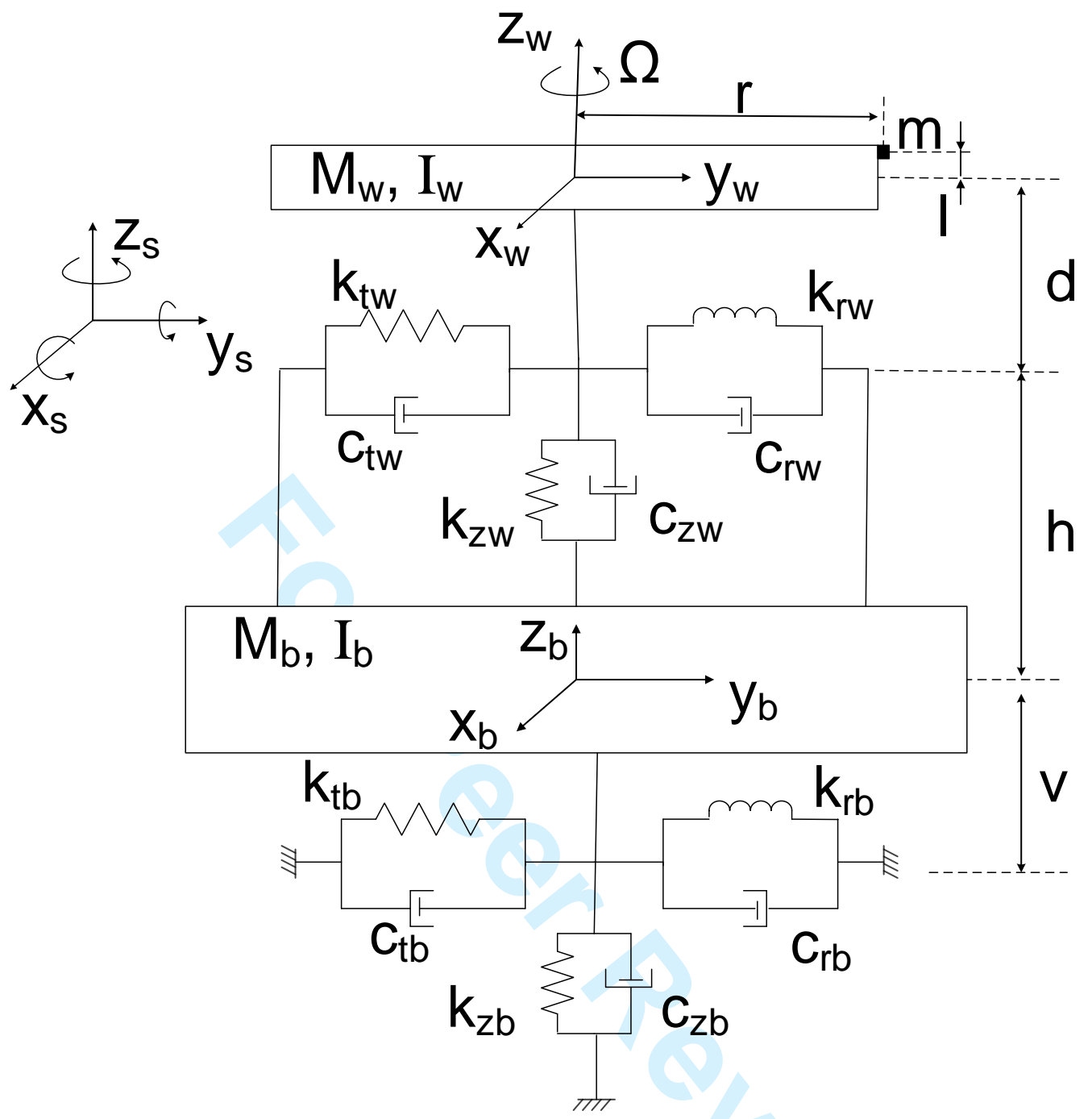

Fig. 1 Simplified imbalance cantilever configured reaction wheel assembly model

operative configurations.

\section{A. Wheel Assembly Modelling}

The development of the complete mathematical model includes two cases: balanced flywheel and imbalanced flywheel. The simplified imbalanced wheel model is shown in Fig. 1. The RWA model consists of a rigid flywheel and wheel-base, with mass and inertia properties, connected via a massless suspension system. In addition, the wheel-base is connected to the ground by means of another suspension system which is able to reproduce the desired boundary condition (i.e. either 
hard-mounted or free-free). The suspension systems were modeled as a combination of five DoFs, including: two combinations of linear springs and dashpots, $k_{t}$ and $c_{t}$ respectively, each in one of the two radial translational DoFs ( $\mathrm{x}$ and $\mathrm{y}$ ); two pairs of torsional springs and dashpots, $k_{r}$ and $c_{r}$ respectively, each in one of the two radial rotational DoFs $(\theta$ and $\varphi)$; a pair of linear spring and dashpot in the axial translational $\operatorname{DoF}(\mathrm{z}), k_{z}$ and $c_{z}$ respectively. The Z-convention is adopted and the RWA is assumed axisymmetric about its shaft. The generalized Lagrangian coordinates in the RWA model are ten (five associated with the flywheel and five describing the motion of the wheel-base): $x_{w}, y_{w}, z_{w}, \theta_{w}, \phi_{w}, x_{b}, y_{b}, z_{b}, \theta_{b}$, and $\varphi_{b}$ whereas torque microvibrations are negligible due to domination of the flywheel steady speed rotation over angular speed perturbation in torque DoF and therefore ignored.

The angular velocity vector, $\boldsymbol{\omega}_{w}$, of the flywheel Centre of Mass (CoM) is defined by means of a Tait-Brian matrix rotation "1-2-3" (where "1" refers to a rotation about the X-axis, $\theta_{w}$, "2" represents the rotation about the y'-axis, $\varphi_{w}$, and " 3 " is the rotation about the z-axis, $\psi_{w}$ ). Due to steady state rotation, it can be assumed $\dot{\psi}_{w}=\Omega$, where $\Omega$ is the constant angular speed of the flywheel. The angular velocity vector in the body coordinate system can be expressed as:

$$
\boldsymbol{\omega}_{w}=\left\{\begin{array}{c}
\dot{\theta}_{w} \cos \psi_{w}+\dot{\varphi}_{w} \cos \theta_{w} \sin \psi_{w} \\
-\dot{\theta}_{w} \sin \psi_{w}+\dot{\varphi}_{w} \cos \theta_{w} \cos \psi_{w} \\
-\dot{\varphi}_{w} \sin \theta_{w}+\Omega
\end{array}\right\}
$$

Considering small angles of rotations and small displacements, the linearized kinetic energy, $T_{w}$, of the balanced flywheel is derived as:

$$
T_{w}=\frac{1}{2}\left[M_{w}\left(\dot{x}_{w}^{2}+\dot{y}_{w}^{2}+\dot{z}_{w}^{2}\right)+I R_{w}\left(\dot{\theta}_{w}^{2}+\dot{\varphi}_{w}^{2}\right)-2 \Omega I Z_{w} \dot{\varphi}_{w} \theta_{w}\right]
$$

Similarly, the linearized kinetic energy, $T_{b}$, of the wheel-base is obtained:

$$
T_{b}=\frac{1}{2}\left[M_{b}\left(\dot{x}_{b}^{2}+\dot{y}_{b}^{2}+\dot{z}_{b}^{2}\right)+I R_{b}\left(\dot{\theta}_{b}^{2}+\dot{\varphi}_{b}^{2}\right)\right]
$$

In order to reproduce the flywheel radial forces and moments due to rotor imperfections, a mass 
imbalance $m$ is added to the flywheel. The point mass is located at a radius $r$ and distance $l$ from the flywheel CoM and produces forces and moments when the flywheel is in operation. It is able to rotate about and move in the three linear DoFs. According to Fig. 1, the position of the imbalance mass with respect to (w.r.t) the inertial frame can be described as:

$$
\boldsymbol{x}_{m}=\left\{\begin{array}{c}
x_{w} \\
y_{w} \\
z_{w}
\end{array}\right\}+\left\{\begin{array}{c}
r\left(-\cos \varphi_{w} \sin \psi_{w}+\sin \varphi_{w} \sin \theta_{w} \cos \psi_{w}\right)+l \sin \varphi_{w} \cos \theta_{w} \\
r\left(\cos \theta_{w} \cos \psi_{w}\right)-l \sin \theta_{w} \\
r\left(\sin \varphi_{w} \sin \psi_{w}+\cos \varphi_{w} \sin \theta_{w} \cos \psi_{w}\right)+l \cos \varphi_{w} \cos \theta_{w}
\end{array}\right\}
$$

Differentiating Eq. (4) w.r.t time, the velocity vector can be computed and finally the kinetic energy associated with the mass imbalance derived:

$$
T_{m}=-m r \Omega^{2}[(1+l) \sin (\Omega t)+(1-l) \cos (\Omega t)]
$$

Assuming:

i. small displacements and angles (to neglect the second order terms),

ii. the mass imbalance is significantly smaller than the mass of the flywheel and

iii. the flywheel angular speed considerably larger than any rotational perturbation velocity about each axis

the kinetic energy related to the RWA imbalance system is given by:

$$
\begin{aligned}
T_{s} & =T_{w}+T_{b}+T_{m} \\
& \approx \frac{1}{2}\left[M_{w}\left(\dot{x}_{w}^{2}+\dot{y}_{w}^{2}+\dot{z}_{w}^{2}\right)+I R_{w}\left(\dot{\theta}_{w}^{2}+\dot{\varphi}_{w}^{2}\right)-2 \Omega I Z_{w} \dot{\varphi}_{w} \theta_{w}\right] \\
& +\frac{1}{2}\left[M_{b}\left(\dot{x}_{b}^{2}+\dot{y}_{b}^{2}+\dot{z}_{b}^{2}\right)+I R_{b}\left(\dot{\theta}_{b}^{2}+\dot{\varphi}_{b}^{2}\right)\right]-m r \Omega^{2}[(1+l) \sin (\Omega t)+(1-l) \cos (\Omega t)]
\end{aligned}
$$

The potential energy for the mass balanced RWA is expressed as function of the internal forces and moments due to the elastic reactions of the suspension systems. The total potential energy is the sum of the translational and rotational potential energies in the four radial DoFs and that 
associated with the axial translational DoF. Due to the connection of the flywheel and the wheelbase through the suspension systems, the relative displacements of the springs are required to derive the potential energy of the system. In addition, no dynamic coupling between axial and radial DoFs is considered and no potential energy is associated with the point mass imbalance.

In relation to Fig. 2, the process to retrieve the stiffness matrix of the RWA is divided in four stages: firstly, the potential energy in the xz-plane $U_{x z}$ is calculated; secondly the potential energy in the yz-plane $U_{y z}$ is evaluated; thirdly, the translational axial potential energy $U_{z}$ is derived.

Finally, the six potential energies, three associated with the flywheel and three associated with the wheel-base, are summed and the linearized potential energy of the system $U_{s}$ is obtained: 


$$
\begin{aligned}
U_{s} & \approx \frac{1}{2}\left[k_{t, w}\left(x_{w}^{2}+y_{w}^{2}+x_{b}^{2}+y_{b}^{2}-2 x_{w} x_{b}-2 y_{w} y_{b}\right)\right. \\
& +\left(k_{t, w} d^{2}+k_{r, w}\right)\left(\theta_{w}^{2}+\varphi_{w}^{2}\right)+\left(k_{t, w} h^{2}+k_{r, w}\right)\left(\theta_{b}^{2}+\varphi_{b}^{2}\right) \\
& +k_{z, w}\left(z_{w}^{2}+z_{b}^{2}-2 z_{w} z_{b}\right)+2 k_{t, w} d\left(y_{w} \theta_{w}-\theta_{w} y_{b}-x_{w} \varphi_{w}+\varphi_{w} x_{b}\right) \\
& \left.+2 k_{t, w} h\left(y_{w} \theta_{b}-\theta_{b} y_{b}-x_{b} \varphi_{b}+\varphi_{b} x_{b}\right)+2\left(h d k_{t, w}-k_{r, w}\right)\left(\theta_{w} \theta_{b}+\varphi_{w} \varphi_{b}\right)\right] \\
& +\frac{1}{2}\left[k_{t, b}\left(x_{b}^{2}+y_{b}^{2}\right)+k_{z, b} z_{b}^{2}+\left(k_{t, b} v^{2}+k_{r, b}\right)\left(\theta_{b}^{2}+\varphi_{b}^{2}\right)+2 v k_{t, b}\left(y_{b} \theta_{b}-x_{b} \varphi_{b}\right)\right]
\end{aligned}
$$

For completion, the work done by the linear and torsional dashpots must be evaluated. Assuming the damping of viscous type, the damping forces and moments generated by the dashpots can be expressed as the product of the viscous damping coefficient and the velocities of the DoFs. According to Fig. 2, the work done can be obtained applying virtual displacements to the flywheel and wheelbase CoMs and subsequently evaluating the forces and moments produced by the dashpots.

Substituting Eq. (6), Eq. (7), and the work done in the Lagrange's equation [36], the linearized EoM of the RWA system in matrix form can be articulated as:

$$
\mathbf{M}_{s} \ddot{\boldsymbol{q}}_{s}+\left(\mathbf{C}_{s}+\mathbf{G}_{s}\right) \dot{\boldsymbol{q}}_{s}+\mathbf{K}_{s} \boldsymbol{q}_{s}=\boldsymbol{f}_{s}
$$

where $\mathbf{M}, \mathbf{C}$ and $\mathbf{K}$ represent the mass, damping and stiffness matrices of the system, respectively, $\mathbf{G}$ includes terms describing the gyroscopic effect, and $\boldsymbol{q}$ is the vector of generalized coordinates. All matrices in Eq. (8) are expanded and illustrated in Appendix A. Note that in both stiffness and damping matrices there are non-zero off-diagonal elements which are representative of the coupled motion of the radial translational and rotational modes due to the RWA cantilever configuration [17]. The terms on the right-hand-side of Eq. (8) represent the excitations in the system due to the imbalance mass. Although Eq. (8) captures the RWA structural modes, the gyroscopic effect, the fundamental harmonics and their amplifications, the model does not include neither suband higher harmonics nor the broadband noise excitations. Parameters for harmonic excitations modelling include the amplitude coefficients $A_{i}$ and harmonics' fractions $H_{i}$, where $i$ is the number of harmonics considered in each DoF. The harmonic fraction value is defined in [37] as function of the ball bearing geometry, described by the internal radius of the cage, $R_{i n t}$, external radius of the 
cage, $R_{\text {ext }}$, and ball radius, $R_{\text {ball }}$. Three different velocities can be quantified: the velocity of the balls and the cage w.r.t. the stator, $\Omega_{\text {cage,stator }}$; the velocity of the balls and the cage w.r.t. the rotor, $\Omega_{\text {cage,rotor }}$; and the velocity of the balls on the two raceways, $\Omega_{\text {ball }}$ :

$$
\Omega_{\text {cage,stator }}=\frac{\Omega R_{e x t}}{R_{\text {int }}+R_{e x t}} \quad \Omega_{\text {cage,rotor }}=\frac{\Omega R_{i n t}}{R_{\text {int }}+R_{e x t}} \quad \Omega_{\text {ball }}=\frac{\Omega R_{\text {int }} R_{e x t}}{R_{\text {ball }}\left(R_{\text {int }}+R_{\text {ext }}\right)}
$$

Broadband random excitations on the other hand are expressed as $W(\Omega)$ at this stage. Liu et al. [38] observed that higher harmonics and broadband noise can be superimposed to fundamental harmonics. Therefore, including these in the excitation vector on the right hand side of Eq. (8), the complete disturbance vector can be obtained:

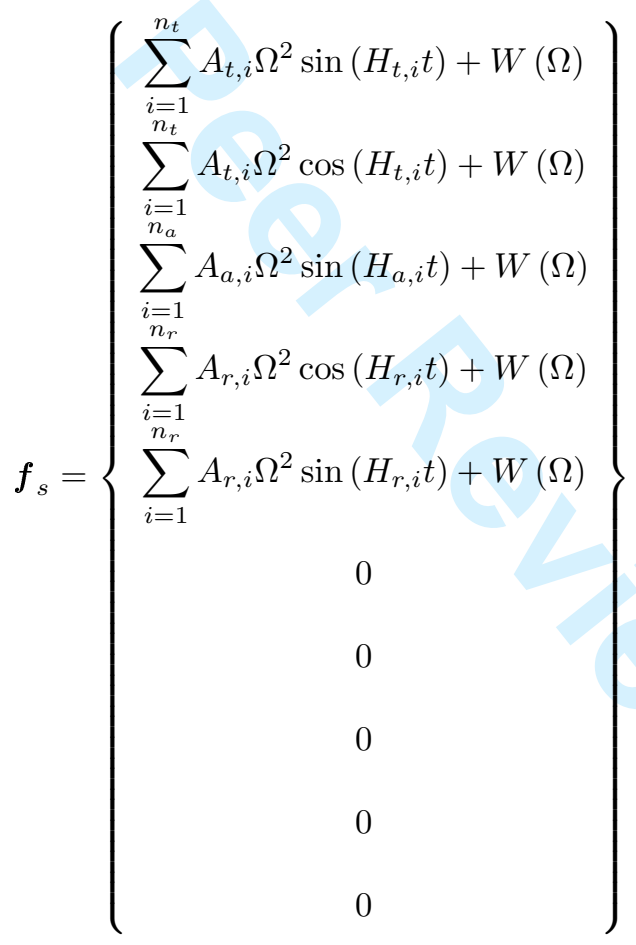

\section{B. Hard-mounted Configuration}

Assuming the suspension system connecting the wheel-base to the ground is extremely rigid, i.e. representative of a hard-mounted boundary condition (also referred to as "blocked" configuration), only five DoFs of the RWA are left in Eq. (8) and the system stiffness and damping matrices reduce to a simpler form. Mathematically, this assumption consists in giving infinite values to the stiffness coefficients of the wheel-base to ground suspension system $\mathbf{K}_{b}$ : 


$$
\lim _{\mathbf{K}_{b} \rightarrow \infty} \mathbf{K}_{s}=\mathbf{K}_{h m}
$$

where $h m$ denotes hard-mounted boundary condition. The resulting system of EoM can be solved to derive the displacement of the flywheel CoM. Finally, the forces and moments at the RWA mounting point can be computed as:

$$
\boldsymbol{f}_{h m}=\mathbf{T}_{h m} \mathbf{K}_{h m} \boldsymbol{x}_{w, h m}
$$

where $\mathbf{T}_{h m}$ is the transformation matrix from the flywheel CoM to the RWA mounting point. Complex coordinates can be used to described the RWA motion and estimate its speed dependent modal frequencies. Assuming small damping and solving the homogenous system, the characteriztic equation can be written as:

$$
\begin{aligned}
\omega_{w}^{4} & -\Omega \frac{I Z_{w}}{I R_{w}} \omega_{w}^{3}-\left(\frac{k_{t, w}}{M_{w}}+\frac{k_{t, w} d^{2}+k_{r, w}}{I R_{w}}\right) \omega_{w}^{2} \\
& +\Omega \frac{k_{t_{w}} I Z_{w}}{M_{w} I R_{w}} \omega_{w}+\frac{k_{t, w} k_{r, w}}{M_{w} I R_{w}}=0 \\
\omega_{z_{w}} & =\sqrt{\frac{k_{z_{w}}}{M_{w}}}
\end{aligned}
$$

Coupling between the two radial and two rotational DoFs can be observed whereas the axial mode is uncoupled and does not change with speed.

\section{Free-free Configuration}

Similarly to the hard-mounted case, the stiffness value of the suspension system connecting wheel-base and ground can be tuned to represent a free-free boundary condition. This is achieved by setting the coefficients with appendix $b$ to zero in both stiffness and damping matrices in Eq. (8).

$$
\lim _{\mathbf{K}_{b} \rightarrow 0} \mathbf{K}_{s}=\mathbf{K}_{\text {fis }}
$$

where fis refers to free-free boundary condition. The resulting system of EoM can be solved to derive the acceleration vectors at the flywheel and wheel-base CoMs. For the purpose of this study, 
we are interested in the accelerations at the mounting point of the RWA. Thereby, a rigid body transformation matrix $\mathbf{T}_{m p}$ has to be applied and the acceleration vector at the RWA mounting point can be computed as:

$$
\ddot{\boldsymbol{x}}_{m p}=\mathbf{T}_{m p} \ddot{\boldsymbol{x}}_{f i s}
$$

where $m p$ denotes the RWA mounting points. The EoM can be re-written in terms of complex coordinates in order to retrieve the structural modes of the RWA. These can be calculated solving the characteriztic polynomial w.r.t $\omega_{s}$ as shown in Eq. (16).

$$
\begin{aligned}
\omega_{s}^{5} & -\Omega \frac{I Z_{w}}{I R_{w}} w_{s}^{4}-\left[\frac{\left(M_{b}+M_{w}\right) k_{t, w}}{M_{b} M_{w}}+\frac{k_{r, w}+k_{t, w} d^{2}}{I R_{w}}+\frac{k_{r, w}+k_{t, w} h^{2}}{I R_{b}}\right] w_{s}^{3} \\
& +\Omega\left[\frac{\left(M_{b}+M_{w}\right) k_{t, w} I Z_{w}}{I R_{w} M_{b} M_{w}}+\frac{\left(k_{r, w}+k_{t, w} h^{2}\right) I Z_{w}}{I R_{b} I R_{w}}\right] \omega_{s}^{2} \\
& +\left[\frac{\left(I R_{b}+I R_{w}\right)\left(M_{b}+M_{w}\right) k_{r, w} k_{t, w}}{I R_{b} I R_{w} M_{b} M_{w}}+\frac{k_{r, w} k_{t, w}(h+d)^{2}}{I R_{b} I R_{w}}\right] \omega_{s} \\
& +\Omega \frac{\left(M_{b}+M_{w}\right) I Z_{w} k_{r, w} k_{t, w}}{I R_{b} I R_{w} M_{b} M_{w}}=0 \\
\omega_{z_{s}} & =\sqrt{k_{z_{w}} \frac{M_{b}+M_{w}}{M_{b} M_{w}}}
\end{aligned}
$$

Likewise the hard-mounted case, the axial mode is decoupled from the remaining DoFs and can be solved independently. On the other hand, the four radial and four rotational DoFs are all coupled together and speed dependent, as expected. The natural frequencies can subsequently be represented as Campbell diagram as function of the angular speed of the flywheel. It can also be noted that if the mass and the inertia of the wheel-base, $M_{b}$ and $I R_{b}$, respectively, tend to infinity (i.e. as to reproduce a hard-mounted boundary condition), Eq. (16) reduces to Eq. (13), as expected.

\section{Dynamic Mass}

The dynamic mass (at times also referred to as apparent mass) is defined as a frequency dependent ratio of the forces imparted on a body and its resulting accelerations measured at the same location where the force is applied. When dealing with rotary mechanisms, the dynamic response also varies with the speed due to the gyroscopic effect. 
Generally speaking, the dynamic mass is a fully populated $6 x 6$ matrix. Earlier works on RWAs have shown that the diagonal coefficients $\left(D_{w_{i i}}\right.$ with $\left.i=1,2,3,4,5,6\right)$ are generally the most important ones $[29,32]$. Moreover, the four cross-DoF terms $D_{w 15}, D_{w 24}, D_{w 42}$, and $D_{w 51}$ have also to be considered, in particular for non-symmetric configurations (i.e. cantilever configured RWA). Concerning the off-diagonal elements (i.e. those correlating the rocking mode of the $\mathrm{x}$ and $\mathrm{y}$ axes), in all previous studies $[17,29,32,33]$, they were assumed negligible and set to zero. However, when a flywheel is in operation, the gyroscopic effect significantly modifies the RWA internal dynamics, and some of the off-diagonal terms assume values (in absolute value) comparable to the other coefficients in the dynamic mass matrix [34]. In this article, a validation of the analytical results reported in [34] is presented. Due to symmetry properties of the dynamic mass about its diagonal and RWA axisymmetry, the dynamic mass matrix can be re-formulated as:

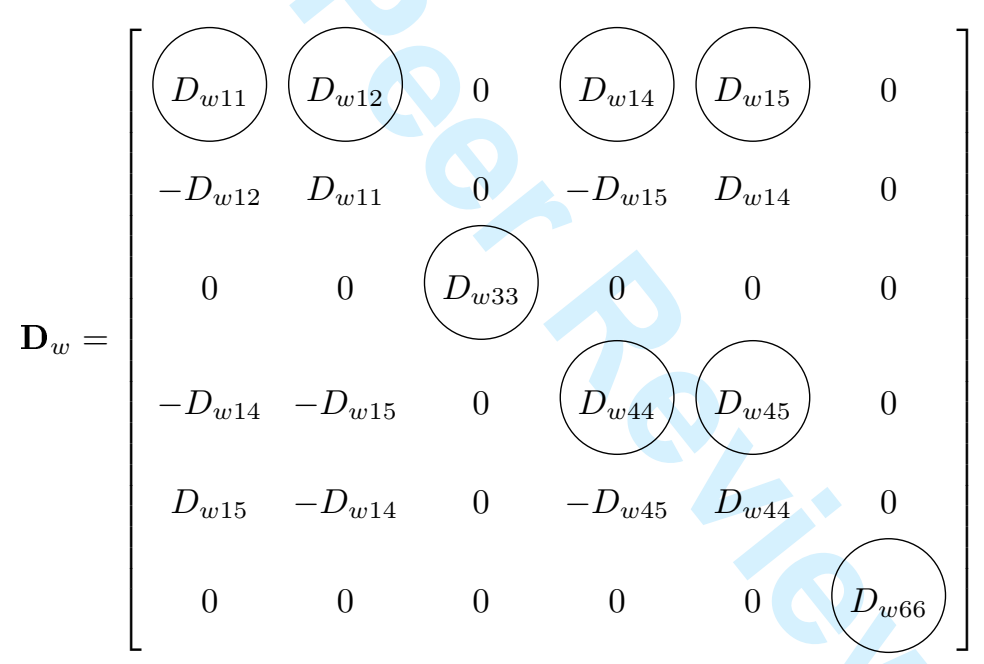

The 36 original elements are now reduced to only 8, circled in Eq. (17), all of which are speed and frequency dependent. As introduced in Section I, a methodology based on measurements of the forces and moments grounding the RWA on a dynamometric platform and the accelerations at the RWA mounting point when this is suspended free-free, is used to retrieve the 8 most significant elements in the RWA dynamic mass matrix. The derivation process involves a re-arrangement of the coupling theory developed in [14] and later expanded in [12, 33]. The coupled loads exchanged at the interface between a source and its supporting structure are function of the inertial properties of the bodies (described by the dynamic mass) and the disturbances generated by the source. In particular, it can be said that the coupled forces and moments can be expressed as a portion of the 
forces and moments measured in a hard-mounted condition:

$$
\boldsymbol{f}_{c}=\boldsymbol{f}_{h m}-\mathbf{D}_{w} \ddot{\boldsymbol{x}}_{c}
$$

where $c$ denotes coupling, and $\boldsymbol{f}_{c}$ and $\ddot{\boldsymbol{x}}_{c}$ are $6 \mathrm{x} 1$ vectors representing the coupled loads and the coupled accelerations, respectively. Nevertheless, when the RWA is suspended free-free but not attached to any supporting structure, the coupled forces vector is null. The coupled accelerations, in contrast, can be re-written in terms of the source free-free accelerations $\ddot{\boldsymbol{x}}_{f i s}$, yielding to:

$$
f_{h m}=\mathbf{D}_{w} \ddot{x}_{f i s}
$$

Equation (19) can be re-formulated in terms of Power Spectral Density (PSD). The dynamic mass is the transfer function between the loads and the accelerations calculated at the same location (i.e. RWA interface mounting point) therefore, when the PSD is applied, its Hermitian (conjugate and transpose) value has to be considered.

$$
\mathbf{\Phi}_{h m}=\mathbf{D}_{w} \mathbf{a}_{f i s} \mathbf{D}_{w}^{H}
$$

Finally, Eq. (20) can be solved for $\mathbf{D}_{w}$ at each speed and each frequency using an iterative process thus to define all the elements in Eq. (17) over the speed and frequency ranges of interest.

\section{RWA Model Validation}

Microvibration measurements were carried out to verify the analytical predictions for both the hard-mounted and free-free boundary conditions. The former involved the use of a dynamometric measurement platform whereas the latter required the specimen to be hung using elastic cords such to reproduce a free-free configuration. Model parameters such as stiffness and natural frequencies were extracted from the test results and compared to the theoretical values. The RWA consisted of a Brass-made rotor, an Aluminum 6082-T6 housing, and a Polyactic acid thermoplastic motor holder (acting as suspension system), resulting in an overall mass of $1.16 \mathrm{~kg}$. A brushless DC-motor was used to run the rotor providing an angular momentum at 4800 revolutions per minute (rpm) 
of $0.553 \mathrm{Nms}$. The RWA was spun from $600 \mathrm{rpm}$ to $4800 \mathrm{rpm}$ with a $60 \mathrm{rpm}$ step increase and the data were recorded for 8 seconds at each speed. The entire test campaign was performed overnight in an extremely quiet environment to maintain the level of the noise as low as possible. In addition, the functioning of electrical devices such as air ventilation was halted. For instance, the typical background noise for axial measurements compared to RWA disturbances generated at $600 \mathrm{rpm}$ is graphed in Fig. 3 for both hard-mounted and free-free boundary conditions.

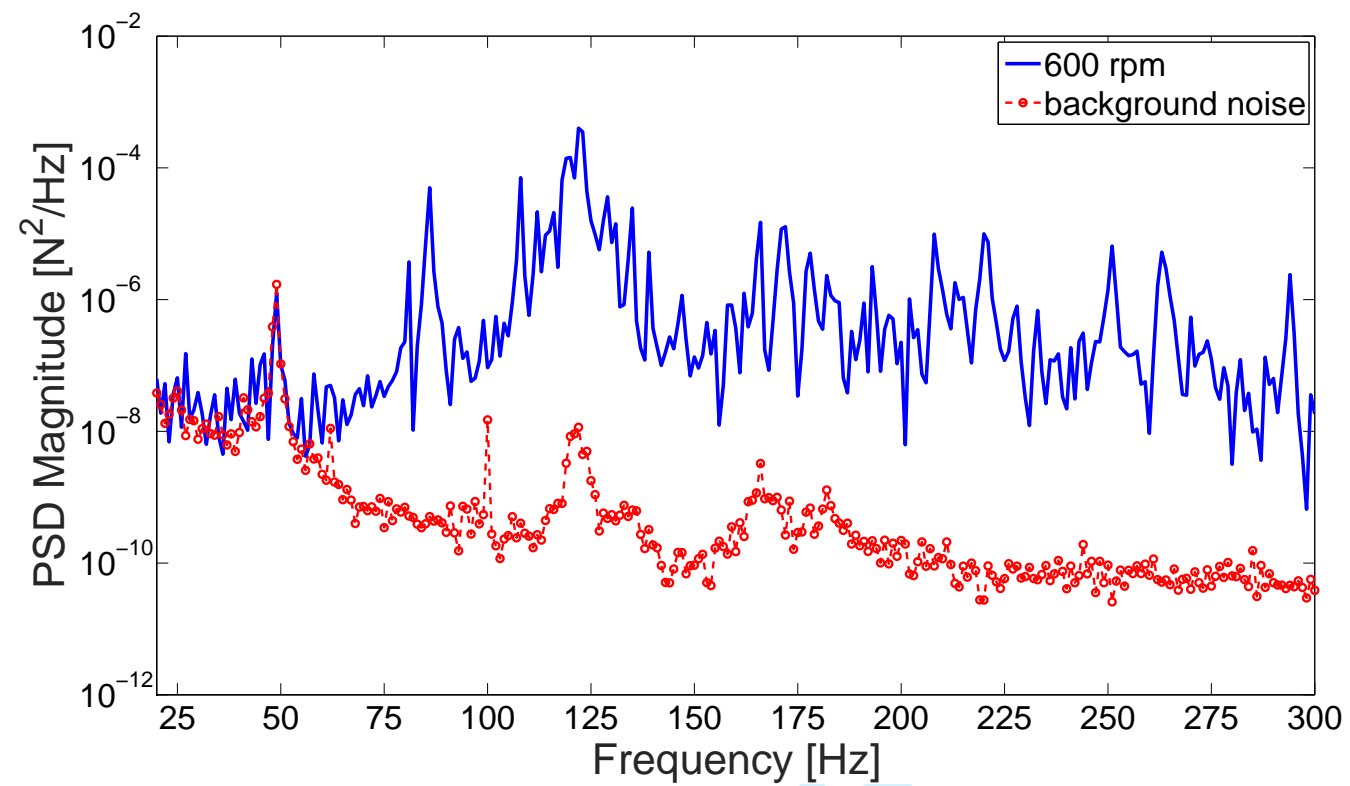

a) Hard-mounted configuration, axial force

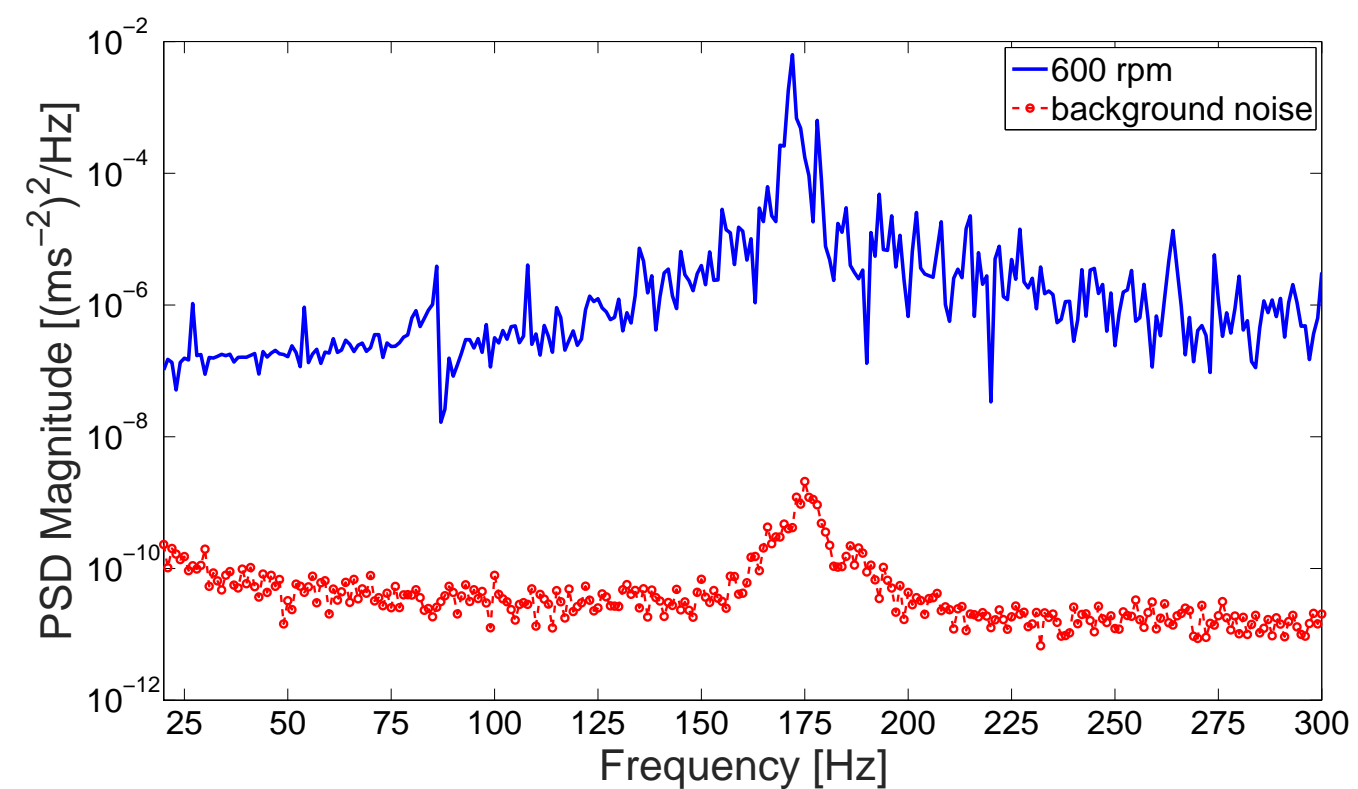

b) Free-free configuration, axial acceleration

Fig. 3 Background noise compared to the excitation level at $600 \mathrm{rpm}$ 


\section{A. Hard-mounted Microvibration Testing}

The hard-mounted RWA-induced disturbances were measured by means of a microvibration measurement platform developed and validated in [31]. A total of three test configurations was needed to achieve the full characterisation of the RWA-induced loads. The measurement setups in the wheel $\mathrm{y}$-axis and z-axis are illustrated in Fig. 4. Measurements along the wheel $\mathrm{x}$-axis were obtained by rotating the RWA y-axis test configuration by 90 degrees anti-clockwise.

In addition, a stripe of high reflective tape (e.g. Kapton tape) was embedded onto the flywheel with the purpose of synchronizing the signals acquired from different measurements (i.e. hardmounted and free-free configurations), facilitating their processing and increasing the reliability of the results.

The predicted speed dependent modal frequencies $\omega_{w}$ obtained from Eq. (13) are plotted as function of the flywheel angular speed and superimposed to the experimental results as red circles, as shown in Fig. 5. Among the four speed dependent modal responses, two are identified as the Forward Whirls (FWs), starting at $51 \mathrm{~Hz}$ and $158 \mathrm{~Hz}$ and increasing with speed, and two represent the Backward Whirls (BWs), starting at $44 \mathrm{~Hz}$ and $154 \mathrm{~Hz}$ decreasing with speed. The axial mode,

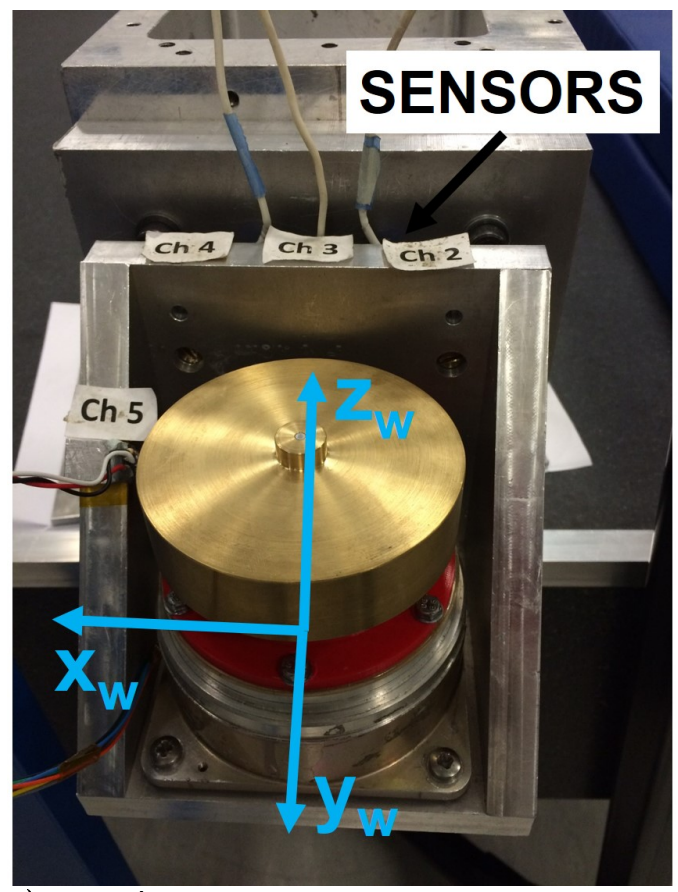

a) $y_{w}$-axis

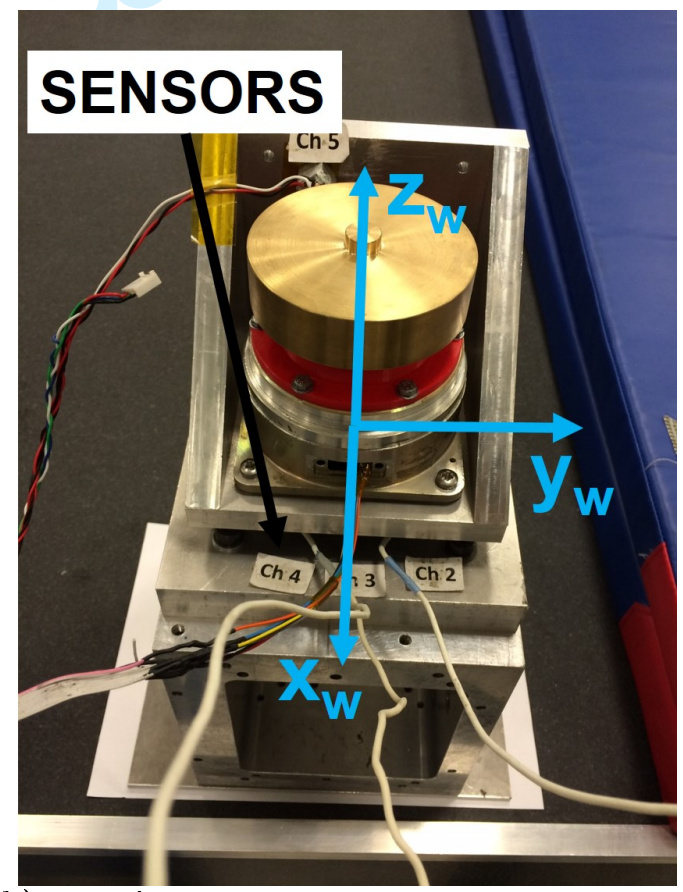

b) $z_{w}$-axis

Fig. 4 Hard-mounted boundary condition test configuration 


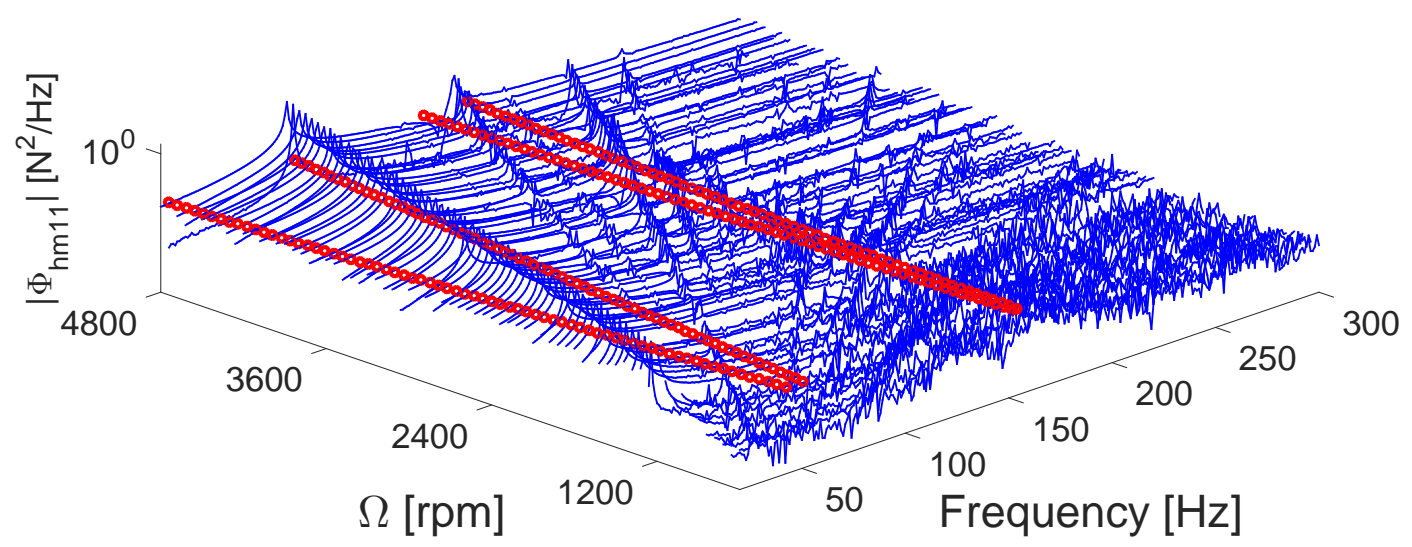

a) PSD of lateral force, $F_{h m, x}$

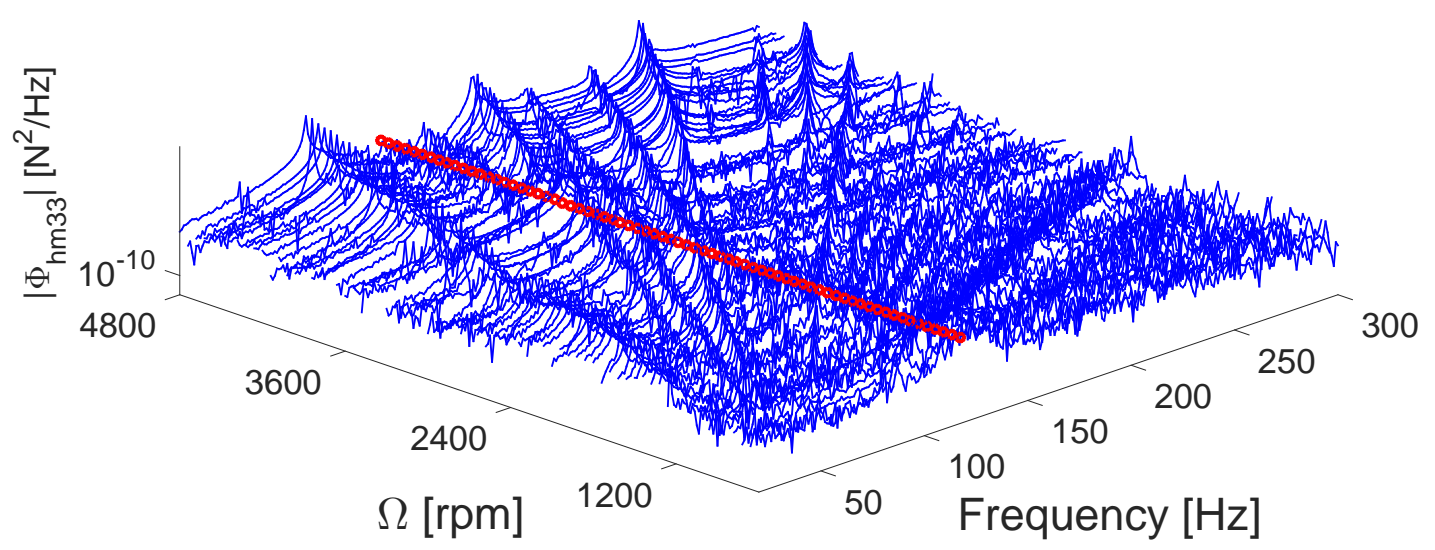

b) PSD of axial force, $F_{h m, z}$

Fig. 5 Hard-mounted loads from the tests superimposed to the RWA predicted structural modes (red circles)

in contrast, is at $117 \mathrm{~Hz}$ and remains constant as speed increases, as expected. A good correlation between the experimental and the simulated results can be observed. In particular, the system displays amplifications due to interaction between the angular speed of the flywheel and the speed dependent modes, matching the theoretical predictions. On this basis, the hard-mounted RWA model was verified and validated.

\section{B. Free-free Microvibration Testing}

The RWA was suspended using elastic cords aiming to reproduce an unconstrained boundary condition. The subsystem was attached to a steel frame which is able to isolate the specimen from 
the surroundings, limiting any external interaction to a minimum. A set of seven accelerometers was installed at the RWA mounting interface. In particular, two accelerometers were placed in the xyplane for measuring the radial translations along $\mathrm{x}$, two accelerometers were set at 90 degrees w.r.t the former accelerometers in the xy-plane allowing measurements of the radial translations along $\mathrm{y}$, and three-accelerometers were placed on top of the interface plate retrieving the axial translations of the RWA, as shown in Fig. 6. The accelerometers' responses were then combined to compute the in- and out-of-plane rotations.

The predicted speed dependent modal frequencies $\omega_{w}$ obtained from Eq. (16) are plotted as function of the flywheel angular speed and superimposed to the experimental results as red circles, as shown in Fig. 7.

Similarly to the hard-mounted case, also here the axial mode is not influenced by speed variations and remains constant at a value of $171 \mathrm{~Hz}$. In addition, two modes corresponding at the FWs start at $75 \mathrm{~Hz}$ and $291 \mathrm{~Hz}$, respectively, and increase with speed whereas the two modes corresponding to the BWs start at $58 \mathrm{~Hz}$ and $290 \mathrm{~Hz}$, respectively, and decrease as speed increases. It can be

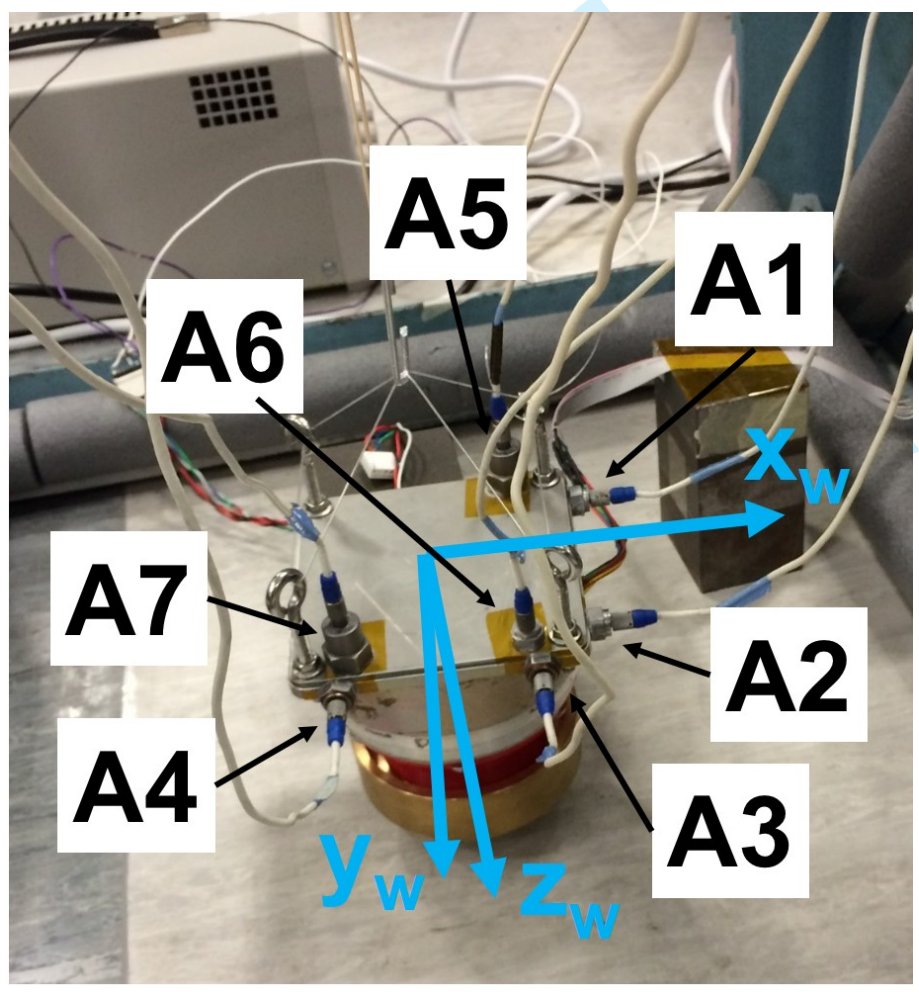

Fig. 6 Free-free boundary condition test configuration 


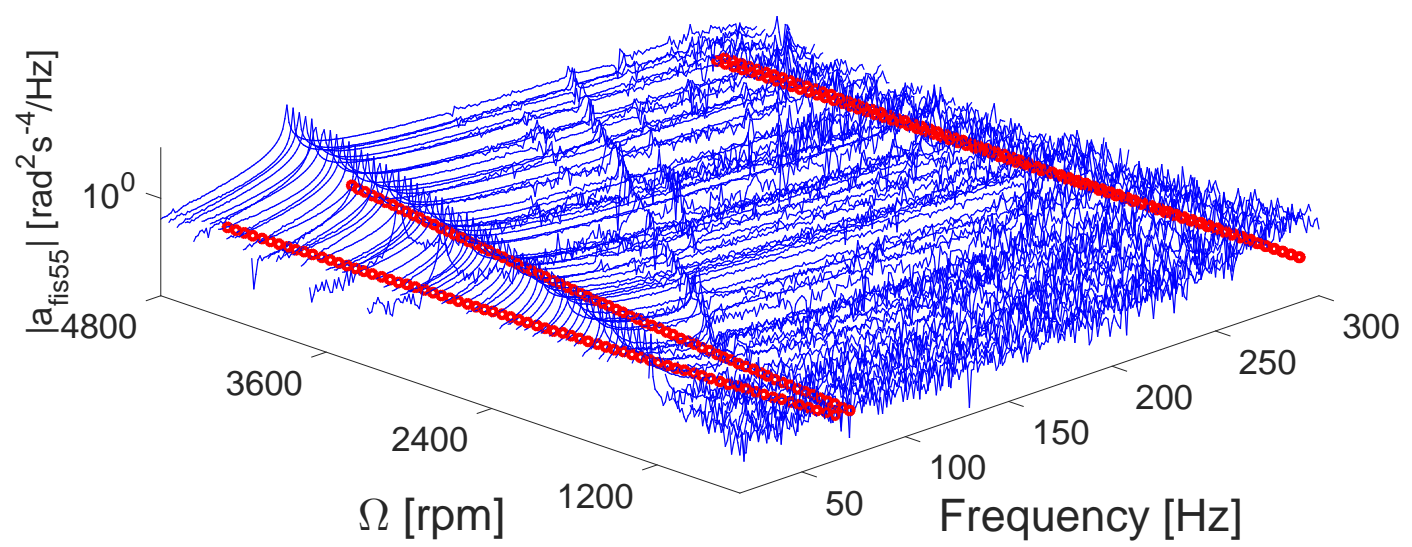

b) PSD of in-plane acceleration, $\ddot{\varphi}_{f i s}$

Fig. 7 Experimental free-free accelerations superimposed to the RWA predicted structural modes (red circles)

observed that amplifications of the system responses occur at values where the angular speed of the flywheel and the speed dependent modes cross, as predicted. Overall, the experimental and analytical outcomes display a good correlation allowing the validation of the free-free RWA model.

\section{Dynamic Mass Measurements and Results}

\section{A. Analytical Prediction}

The importance to evaluate the effect of the gyroscopic effect on the dynamic mass when the RWA is in operation (i.e. flywheel spinning at speed different from zero) can be observed comparing the RWA theoretical system response in either static or dynamic configurations. 


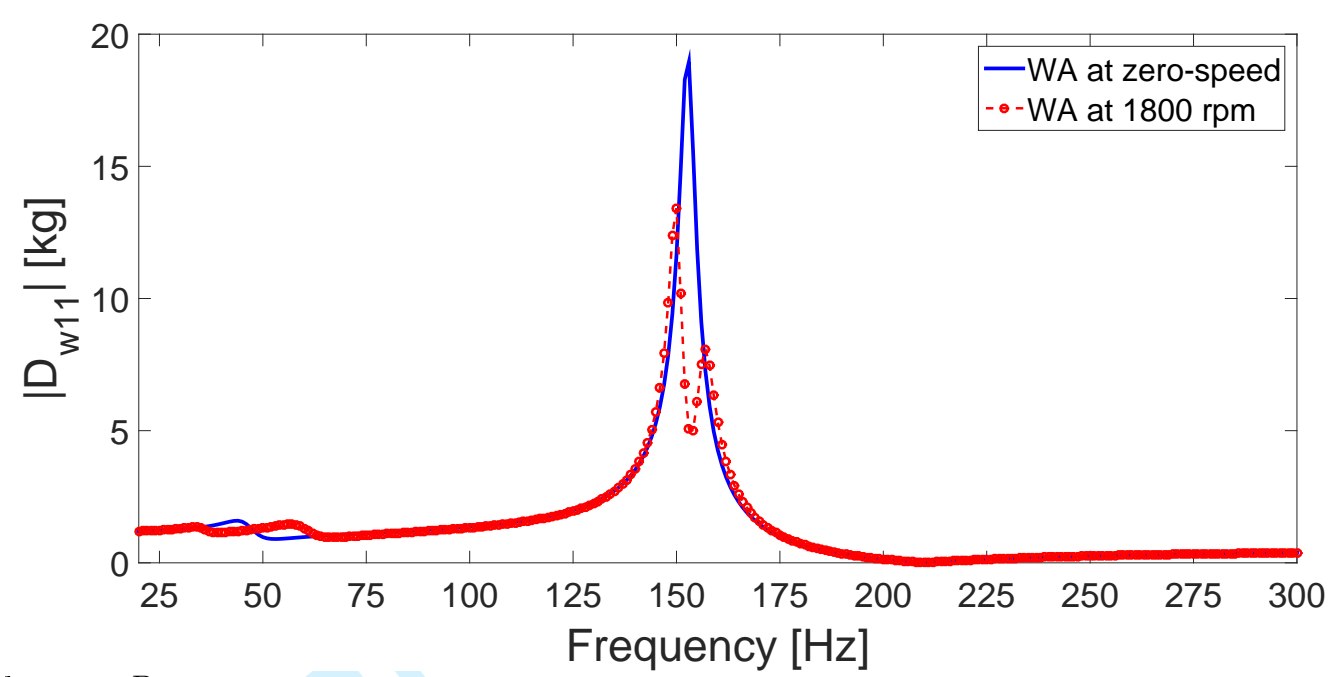

a) element $D_{w 11}$

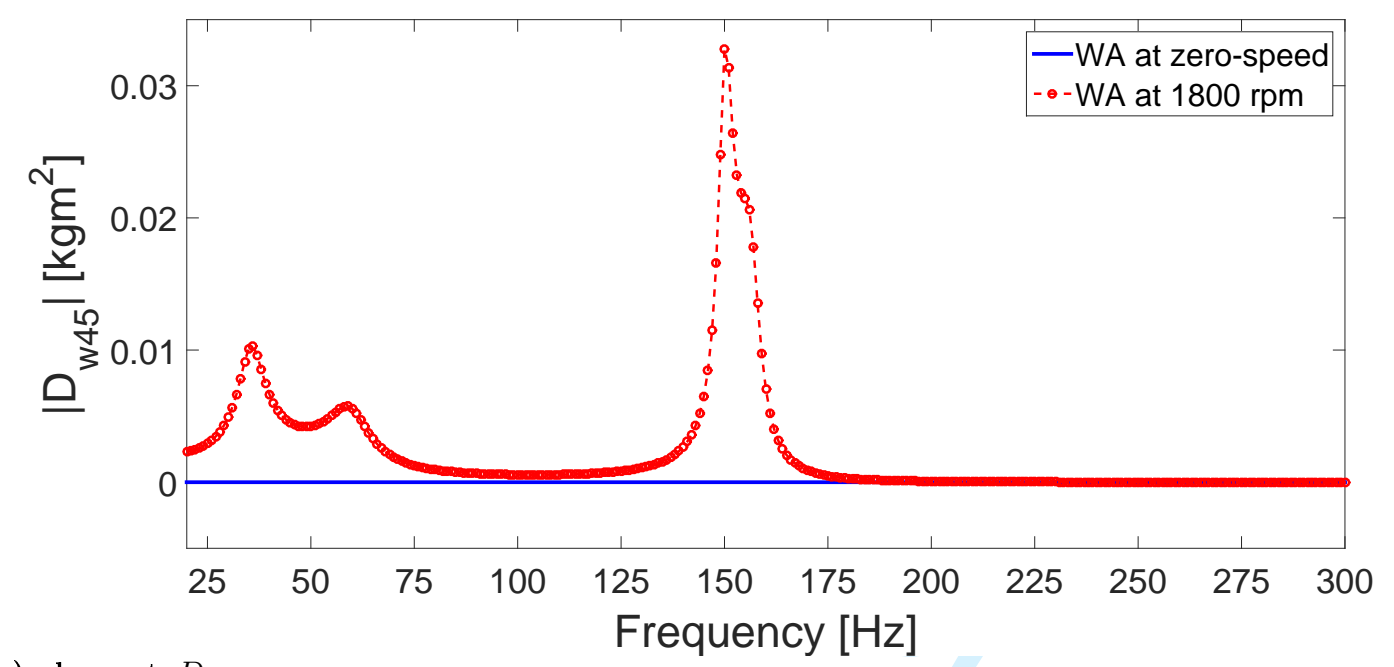

b) element $D_{w 45}$

Fig. 8 Comparison between static and in-operation dynamic mass

Figure 8 highlights the main differences and, in particular, shows how the two initial structural resonances split in four modal resonances due to the gyroscopic effect. In addition, some of the offdiagonal terms, which are null in the static configuration, are no longer negligible when the RWA is in operation and, therefore, must be included for the complete characterization of the RWA. It is worth to note that a linear scale, rather than a logarithmic one, has been used here to show the line representing the analytical response when the flywheel is not operating in Fig. 8b due to its zero constant value throughout the whole range of frequency.

For simplicity only the elements $D_{w 11}, D_{w 33}$, and $D_{w 45}$ are shown in Fig. 9. The dynamic mass 


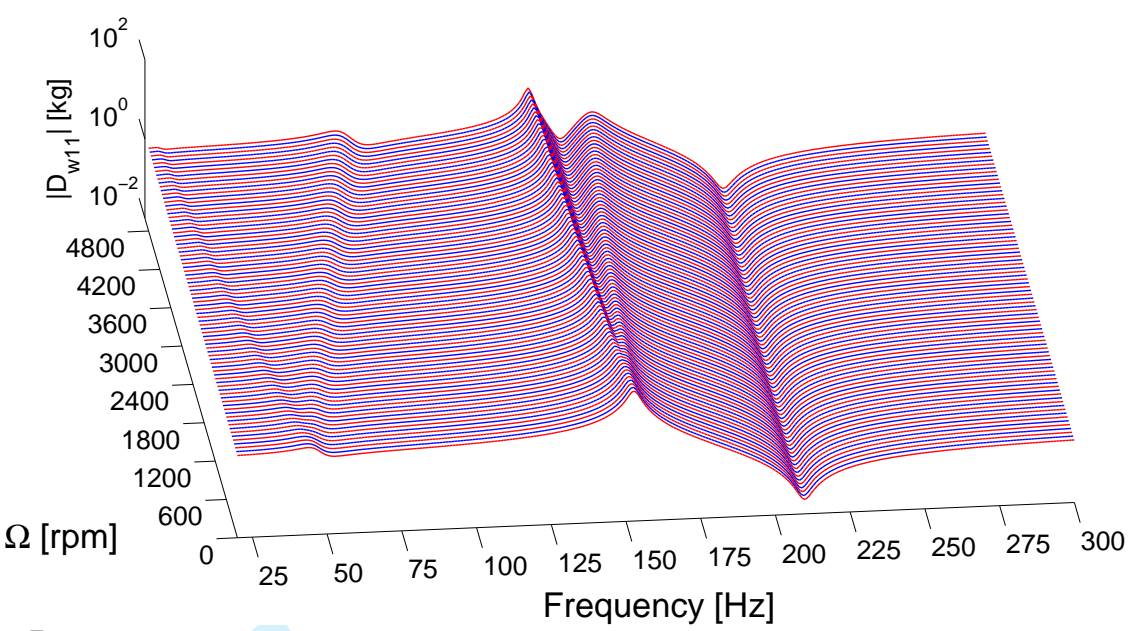

a) element $D_{w 11}$

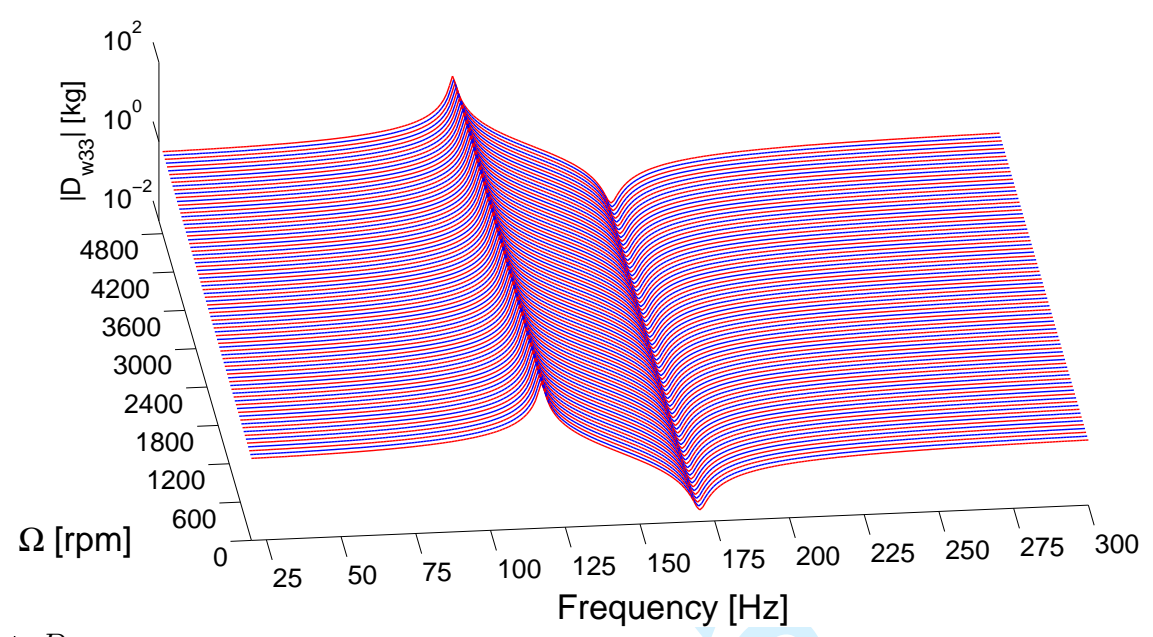

b) element $D_{w 33}$

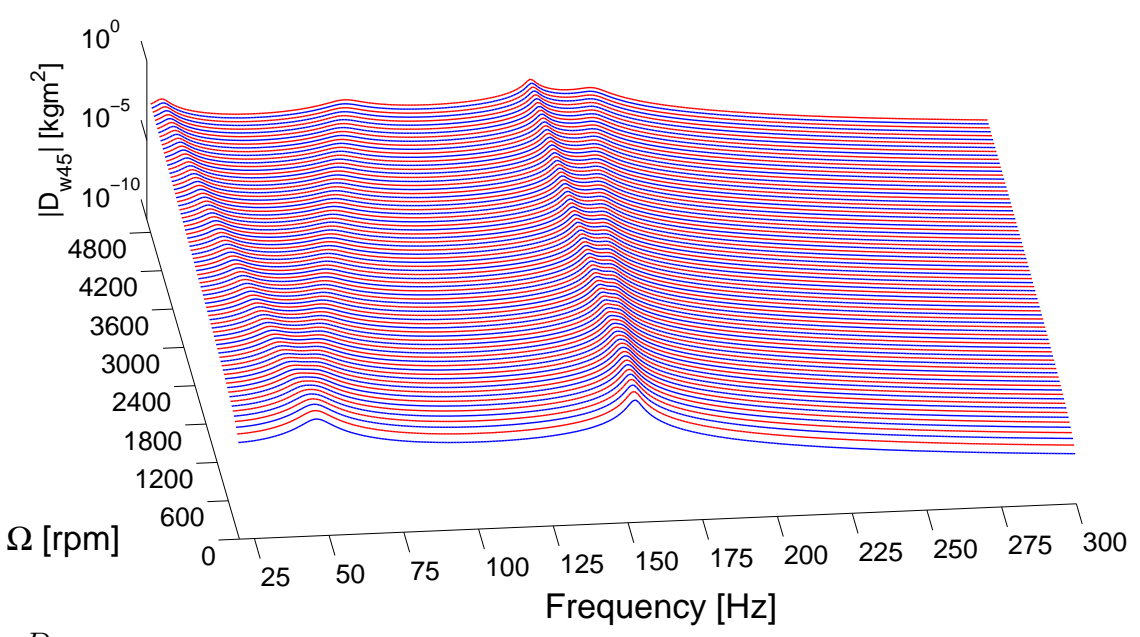

c) element $D_{w 45}$

Fig. 9 Predicted dynamic mass behaviour as function of frequency and flywheel angular speed 
elements are plotted in a range of frequency from 20 to $300 \mathrm{~Hz}$ and in a range of flywheel speed from 600 to $4800 \mathrm{rpm}$ with $60 \mathrm{rpm}$ interval. The axial mode remains constant as speed increases, as expected, whereas all the other coefficients display a strong dependance from both frequency and speed. Moreover, they present coupled effects between each DoF whereas the axial mode remains uncoupled, typical of cantilever-configured RWAs.

\section{B. Experimental results}

The outcomes derived in section III were combined to evaluate the speed and frequency dependent dynamic mass of the RWA. Due to the dynamic mass imaginary complex nature (intended as composed by a real and an imaginary number) and therefore, the importance of the phase between the signals, a synchronisation procedure was required before their direct implementation in Eq. (20). This was accomplished by means of a reflective tape (e.g. Kapton tape) on the flywheel and an optical sensor which recorded any passage during the tests. The optical sensor signals measured in both the hard-mounted and the free-free tests were then cross-correlated and subsequently opportunely shifted to obtain the desired synchronisation. An example of the optical sensor response for the flywheel spinning at $600 \mathrm{rpm}$ during the hard-mounted test is presented in Fig. 10.

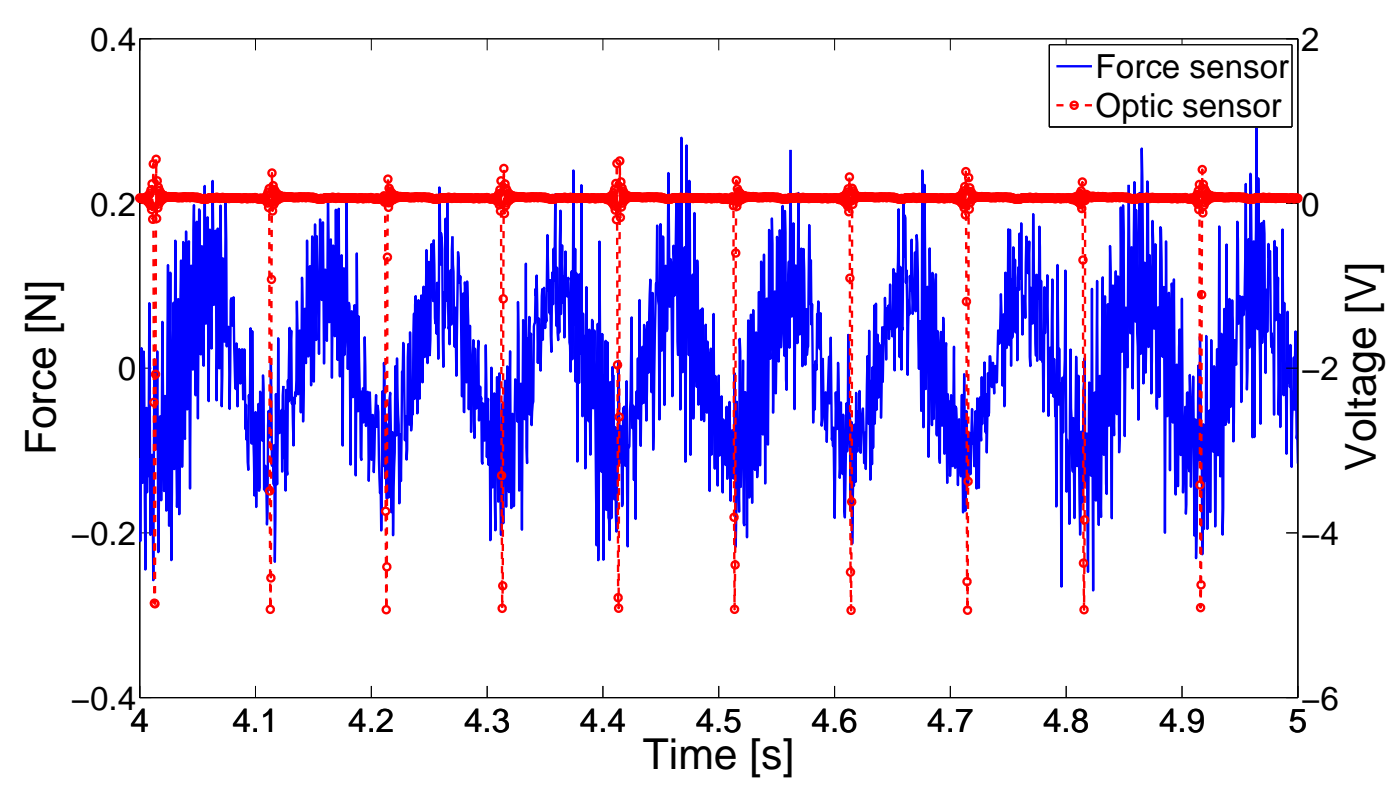

Fig. 10 Optical sensor signal superimposed to a force transducer response as the flywheel spins at $600 \mathrm{rpm}$. 
The synchronised loads and accelerations were post-processed and reformulated in terms of PSD and cross-PSD to form two $6 \mathrm{x} 6$ complex matrices, $\boldsymbol{\Phi}_{h m}$ and $\mathbf{a}_{f i s}$. The off-diagonal elements of the forces' and accelerations' matrices $\Phi_{h m, j i}$ and $a_{f i s, j i}$, (with $i \neq j$ ), respectively, are the conjugate of the elements $\Phi_{h m, i j}$ and $a_{f i s, i j}$. The matrices were subsequently implemented in Eq. (20) to retrieve the dynamic mass matrix.

The equation was solved for each frequency of interest and for each angular speed, allowing the full description of the RWA dynamic response. It must be observed that due to the non linear nature of the system (72 equations of the second order, 36 for the real part and 36 for the imaginary part, and 16 unknowns, 8 real and 8 imaginary), for the system to converge to an acceptable solution, a reasonable estimate of the initial value of the coefficients circled in Eq. (17) was mandatory. In particular, the knowledge of the main diagonal elements (e.g. the total mass of the RWA and the second moment of inertia calculated at the RWA mounting interface)allows to achieve accurate results. In practice, these are also the simplest values that can be measured (i.e. by means of a scale). For the RWA under investigation these are $1.16 \mathrm{~kg}$ and $5.9 \times 10^{-3} \mathrm{kgm}^{-2}$, respectively. The outcomes of the analysis were subsequently compared to the theoretical predictions and are here illustrated in Fig. 11 for an angular speed equal to $660 \mathrm{rpm}$.

An overall acceptable agreement can be observed, in particular the axial translation mode is correctly captured throughout the frequency range. The experimental results display an initial poor correlation (between $20 \mathrm{~Hz}$ and $50 \mathrm{~Hz}$ ) for the radial mode in Fig. 11b. Despite the trend of the curve is similar to the predicted one, its amplitude is lower. Nevertheless, the outcomes become more accurate as frequency increases, specially after the first backward and forward whirls. Moving towards the second backward and forward whirls, these show a better agreement with the theoretical values. The curve then decreases with the same pattern of the FE model estimate. However, a series of spikes between $230 \mathrm{~Hz}$ and $260 \mathrm{~Hz}$ can be observed which are not present in the analytical prediction. Considering the axial mode only, the experimental and predicted data correlate well in all the range of frequency and the modal resonance at $117 \mathrm{~Hz}$ is correctly captured.

The mismatches occurring at both low and high frequencies were thoroughly investigated as to determine the causes which led to values differing from the predicted ones. The dominant reason was 


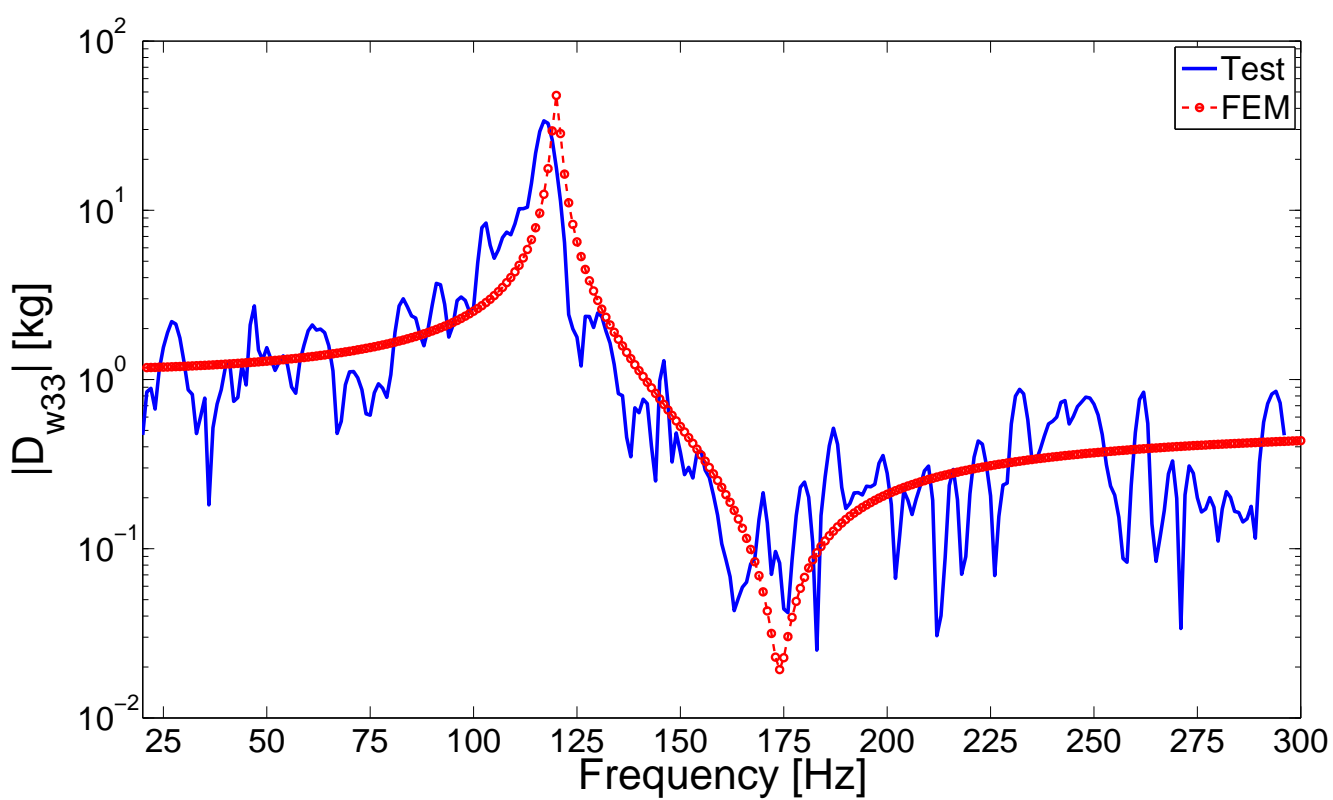

a) element $D_{w 33}$

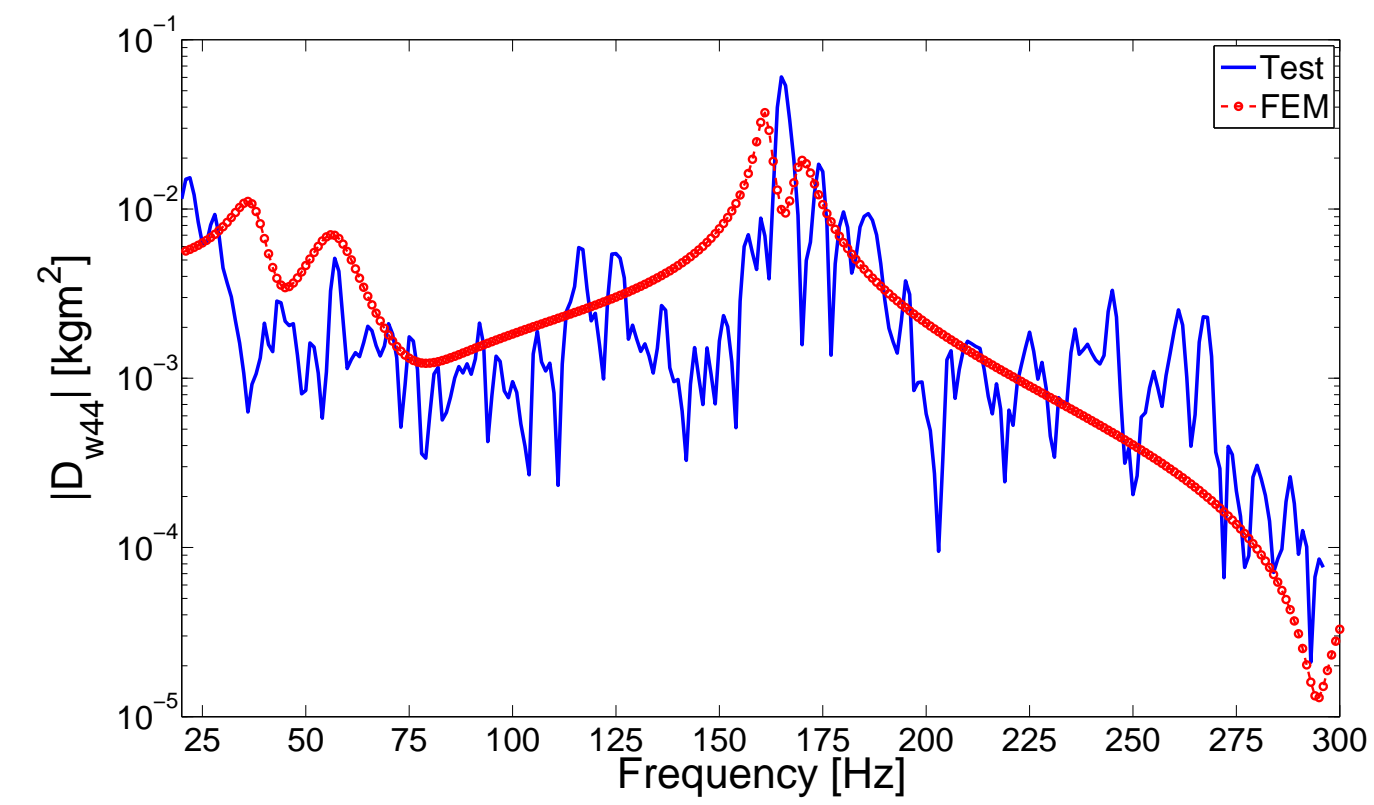

b) element $D_{w 44}$

Fig. 11 Comparison between predicted and experimental dynamic mass at $1320 \mathrm{rpm}$

identified in the different characteriztics of the broadband noises, in terms of spectral contribution, measured in the hard-mounted and free-free configurations. In section IV C, a demonstration of the influence of the broadband noise is given by means of the analytical model. The outcomes are then used against test results to validate the aforementioned thesis. 


\section{Influence of broadband noise}

The noise spectra measured during the two test campaigns (hard-mounted and free-free configurations) may severely affect the convergence of Eq. (20). If the spectral contribution of the noise obtained in the two different configurations is significantly different (hence the RWA excitation vectors are different) then the iterative process to calculate the dynamic mass of the RWA using Eq. (20) may fail or lead to inaccurate calculations at some frequencies in the spectrum. This matter was investigated analytically using the model described in section II and the outcomes are shown in Fig. 12.

A set of random noise signals was generated and applied to the models. A noise ratio was calculated between the magnitude of the noise applied to the model in hard-mounted boundary condition and that given as input to the model in free-free configuration. Due to the nature of this analysis, for which only the effect of the background noise is emulated, a zero-speed condition was assumed. The results displayed a remarkable difference as the noise ratio increases. It can also be observed that the noise ratio has less influence on the axial DoF compared to the other DoFs. Moreover, the variation of dynamic mass coefficient $D_{w 33}$ as function of the noise ratio is linear. Furthermore, Fig. 12b shows that even small differences in the noise spectra in the range between $270 \mathrm{~Hz}$ and $300 \mathrm{~Hz}$ may lead to considerable miscalculations in the dynamic mass estimation.

The spectral contribution of the noise must thereby be similar between the hard-mounted case and the free-free case. The background noise spectrum derived from the hard-mounted configuration was compared to that obtained in the free-free configuration. The latter required to be re-elaborated using Eq. (20), where the term $\mathbf{a}_{\text {fis }}$ represents the PSD of the background noise in the free-free boundary condition and the term $\mathbf{D}_{w}$ represents the RWA dynamic mass from FE model. The outcomes are shown in Fig. 13.

It can be observed that the spectral contribution is different in the range of frequency between $20 \mathrm{~Hz}$ and $50 \mathrm{~Hz}$ and above $230 \mathrm{~Hz}$ between the two configurations. This therefore, proves that the dominant reason which led to a inaccurate calculation of the dynamic mass in the aforementioned ranges of frequency, as depicted in Fig. 11, lies in the different noise spectra between the hardmounted and the free-free tests, validating the thesis herein stated. 


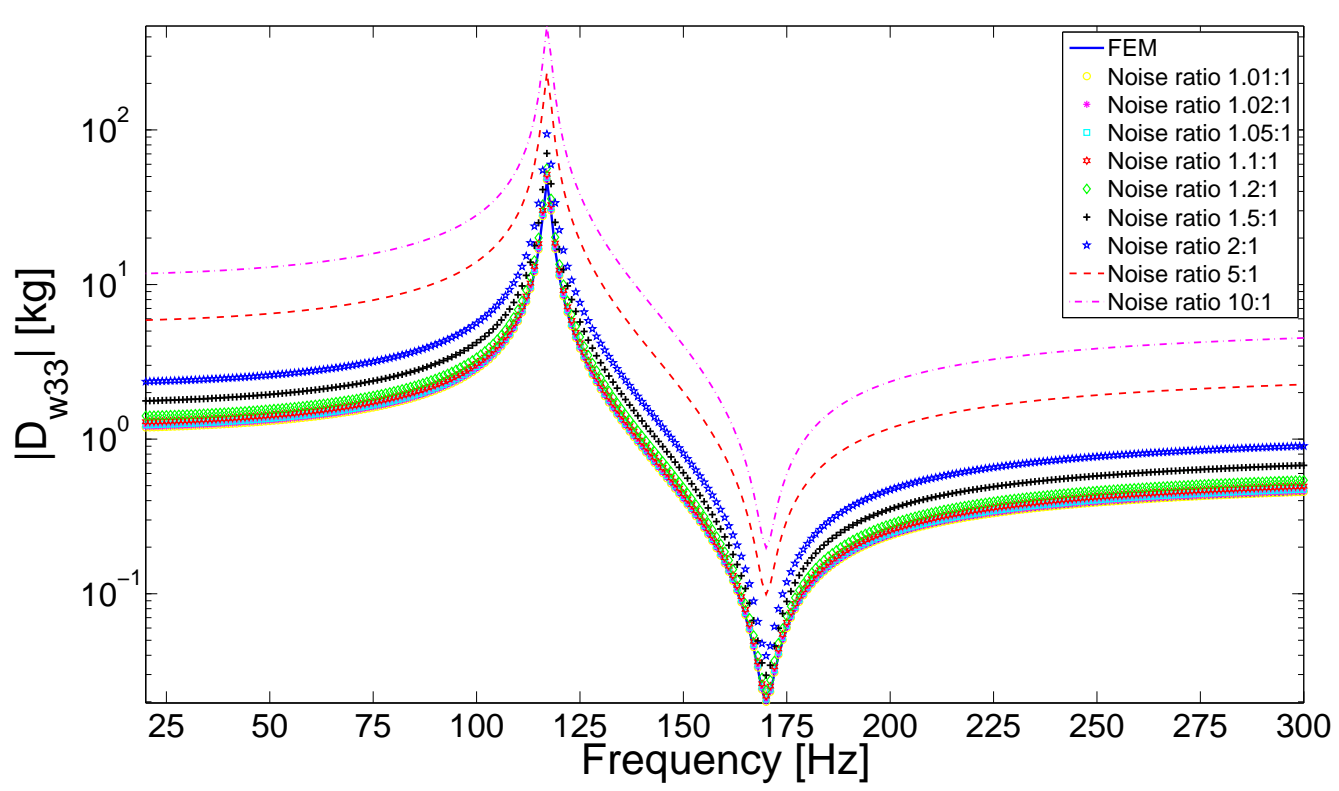

a) element $D_{w 33}$

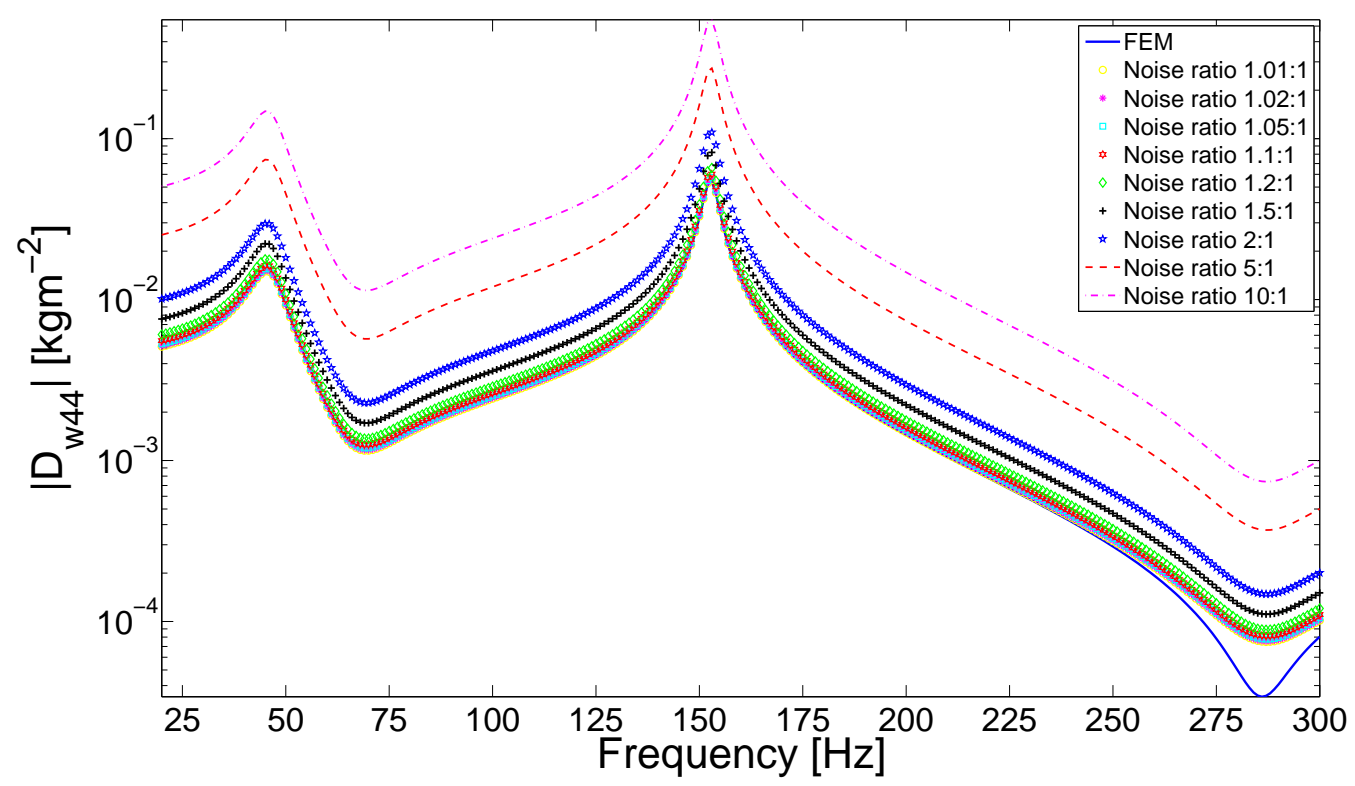

b) element $D_{w 44}$

Fig. 12 Effect of different noise inputs on the calculation of the wheel assembly dynamic mass

\section{Conclusions}

In this paper, a mathematical model able to characterize the microvibrations produced by a mass imbalanced cantilever-configured RWA in both hard-mounted and free-free boundary conditions, including the gyroscopic effect, has been developed and validated. This work also presents a novel 


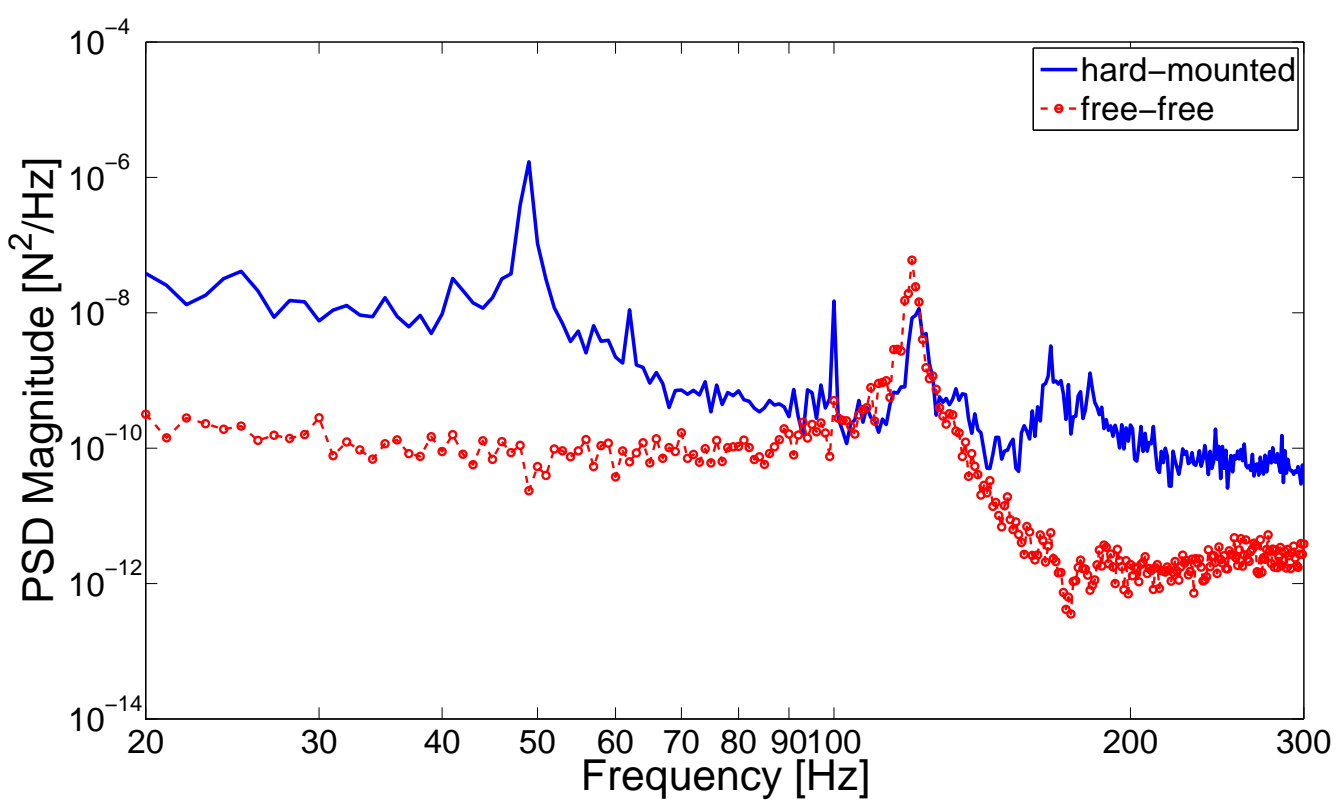

a) element $\Phi_{h m 33}$

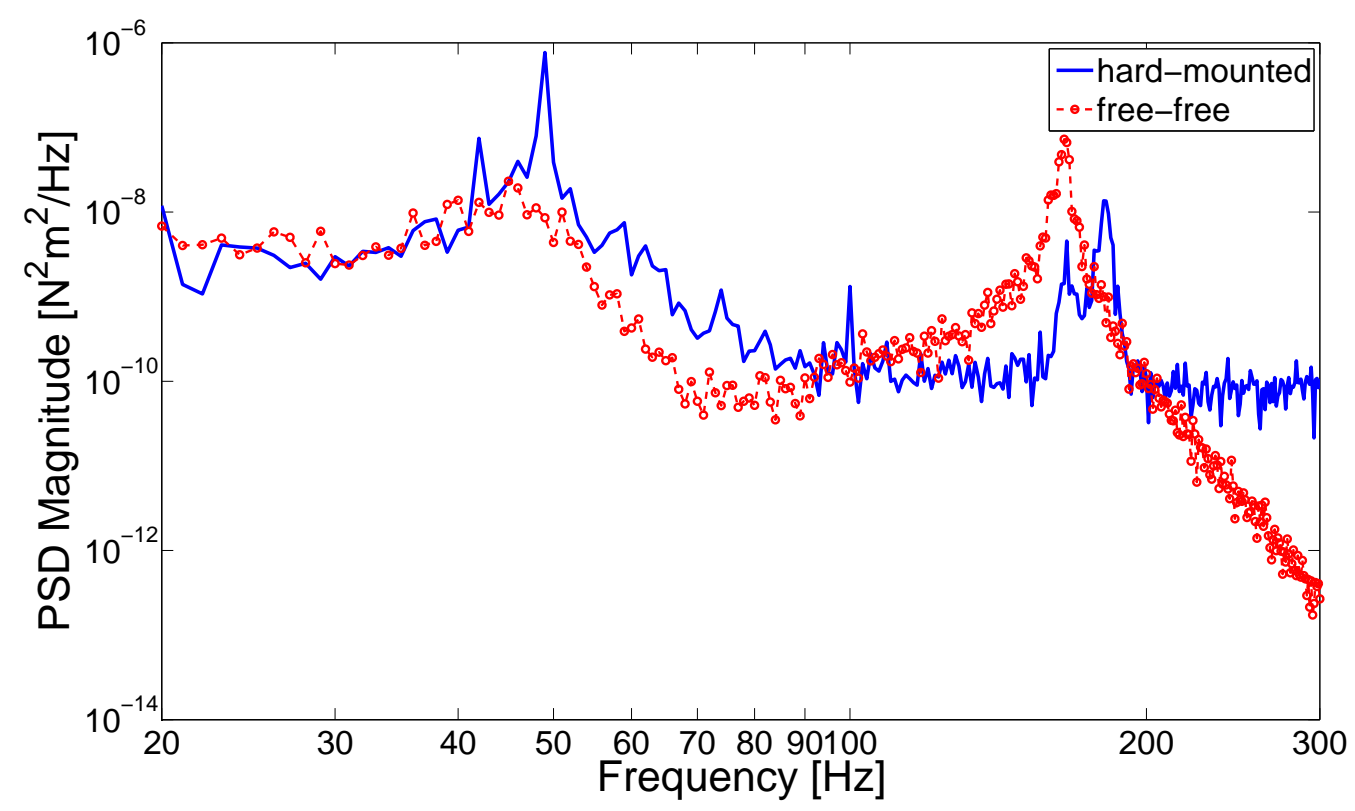

b) element $\Phi_{h m 44}$

Fig. 13 Spectral contribution of the background noise in the hard-mounted and free-free configurations

methodology for the measurement of the dynamic mass, which is derived from a re-elaboration of the coupling theory. This represents an alternative approach to the current practice which involved the use of mini-shakers in a challenging test setup (e.g. free-free suspended RWA with the flywheel spinning while forces are exerted by mini-shakers). The procedure presented herein has proved to 
produce accurate outcomes implementing an extremely simpler test configuration. This involves the use of the reaction loads at the RWA mounting interface measured in a hard-mounted configuration and the accelerations at the RWA mounting interface measured in a free-free boundary condition. In addition, compared to the traditional approach (where the flywheel is not in operation), the method allows the complete characterization of the RWA and express the dynamic mass as function of both frequency and flywheel angular speed. A good agreement was observed between the mathematical model and the experimental data and the forward and backward whirls due to the gyroscopic effect were correctly captured. The main differences between the static and in-operation dynamic mass were highlighted, showing the significant influence of the angular speed on the RWA dynamic response. In addition, it was observed that the off-diagonal terms typically neglected in the previous approaches, become important when the flywheel is spinning and, therefore, require to be included for the complete characterization of microvibration sources. Finally, a brief investigation on the influence of the noise on the derivation of the dynamic mass using the novel approach was conducted. Different set of noise signals were implemented in both the hard-mounted and free-free analytical models. The outcomes showed that different levels of noise between hard-mounted and free-free tests can significantly deteriorate the quality of the results.

\section{Appendix}

With reference to Eq. (8), all matrices are as follows:

$$
\begin{gathered}
\boldsymbol{q}_{s}=\left\{x_{w}, y_{w}, z_{w}, \theta_{w}, \varphi_{w}, x_{b}, y_{b}, z_{b}, \theta_{b}, \varphi_{b}\right\}^{T} \\
\mathbf{M}_{s}=\operatorname{diag}\left\{M_{w}, M_{w}, M_{w}, I R_{w}, I R_{w}, M_{b}, M_{b}, M_{b}, I R_{b}, I R_{b}\right\} \\
\mathbf{G}_{s}=\left\{\begin{array}{l}
G_{s 45}=\Omega I Z_{w} \\
G_{s 54}=-\Omega I Z_{w} \\
0, \quad \text { elsewhere }
\end{array}\right.
\end{gathered}
$$


Table 1 Damping coefficients in compact format

\begin{tabular}{ll}
\hline$c_{11}=c_{t, w}$ & $c_{15}=-c_{t, w} d$ \\
$c_{33}=c_{z, w}$ & $c_{44}=c_{t, w} d^{2}+c_{r, w}$ \\
$c_{29}=c_{t, w} h$ & $c_{49}=c_{t, w} h d-c_{r, w}$ \\
$c_{66}=c_{t, w}+c_{t, b}$ & $c_{88}=c_{z, w}+c_{z, b}$ \\
$c_{79}=-\left(c_{t, w} h-c_{t, b} v\right)$ & $c_{99}=c_{t, w} h^{2}+c_{r, w}+c_{t, b} v^{2}+c_{r_{v}}$ \\
\hline
\end{tabular}

$$
\mathbf{C}_{s}=\left[\begin{array}{cccccccccc}
c_{11} & 0 & 0 & 0 & c_{15} & -c_{11} & 0 & 0 & 0 & -c_{29} \\
0 & c_{11} & 0 & -c_{15} & 0 & 0 & -c_{11} & 0 & c_{29} & 0 \\
0 & 0 & c_{33} & 0 & 0 & 0 & 0 & -c_{33} & 0 & 0 \\
0 & -c_{15} & 0 & c_{44} & 0 & 0 & c_{15} & 0 & c_{49} & 0 \\
c_{15} & 0 & 0 & 0 & c_{44} & -c_{15} & 0 & 0 & 0 & c_{49} \\
-c_{11} & 0 & 0 & 0 & -c_{15} & c_{66} & 0 & 0 & 0 & -c_{79} \\
0 & -c_{11} & 0 & c_{15} & 0 & 0 & c_{66} & 0 & c_{79} & 0 \\
0 & 0 & -c_{33} & 0 & 0 & 0 & 0 & c_{88} & 0 & 0 \\
0 & c_{29} & 0 & c_{49} & 0 & 0 & c_{79} & 0 & c_{99} & 0 \\
-c_{29} & 0 & 0 & 0 & c_{49} & -c_{79} & 0 & 0 & 0 & c_{99}
\end{array}\right]
$$

Table 2 Stiffness coefficients in compact format

\begin{tabular}{ll}
\hline$k_{11}=k_{t, w}$ & $k_{15}=-k_{t, w} d$ \\
$k_{33}=k_{z, w}$ & $k_{44}=k_{t, w} d^{2}+k_{r, w}$ \\
$k_{29}=k_{t, w} h$ & $k_{49}=k_{t, w} h d-k_{r, w}$ \\
$k_{66}=k_{t, w}+k_{t, b}$ & $k_{88}=k_{z, w}+k_{z, b}$ \\
$k_{79}=-\left(k_{t, w} h-k_{t, b} v\right)$ & $k_{99}=k_{t, w} h^{2}+k_{r, w}+k_{t, b} v^{2}+k_{r_{v}}$ \\
\hline
\end{tabular}




$$
\mathbf{K}_{s}=\left[\begin{array}{cccccccccc}
k_{11} & 0 & 0 & 0 & k_{15} & -k_{11} & 0 & 0 & 0 & -k_{29} \\
0 & k_{11} & 0 & -k_{15} & 0 & 0 & -k_{11} & 0 & k_{29} & 0 \\
0 & 0 & k_{33} & 0 & 0 & 0 & 0 & -k_{33} & 0 & 0 \\
0 & -k_{15} & 0 & k_{44} & 0 & 0 & k_{15} & 0 & k_{49} & 0 \\
k_{15} & 0 & 0 & 0 & k_{44} & -k_{15} & 0 & 0 & 0 & k_{49} \\
-k_{11} & 0 & 0 & 0 & -k_{15} & k_{66} & 0 & 0 & 0 & -k_{79} \\
0 & -k_{11} & 0 & k_{15} & 0 & 0 & k_{66} & 0 & k_{79} & 0 \\
0 & 0 & -k_{33} & 0 & 0 & 0 & 0 & k_{88} & 0 & 0 \\
0 & k_{29} & 0 & k_{49} & 0 & 0 & k_{79} & 0 & k_{99} & 0 \\
-k_{29} & 0 & 0 & 0 & k_{49} & -k_{79} & 0 & 0 & 0 & k_{99}
\end{array}\right]
$$

\section{References}

[1] Hyde, T. T., Ha, K. Q., Johnston, J. D., Howard, J. M., and Mosier, G. E., "Integrated Modeling Activities for the James Webb Space Telescope: Optical Jitter Analysis," SPIE Conference on Astronomical Telescopes and Instrumentation, Glasgow, Scotland, 2004

[2] Katsukawa, Y., Masada, Y., Shimizu, T., Sakai, S., and Ichimoto, K., "Pointing Stability of Hinode and Requirements for the Next Solar Mission Solar-C," International Conference on Space Optics , Rhodes, Greece, 2010

[3] Remedia, M., Aglietti, G.S., and Richardson, G., "A Stochastic Methodology for Predictions of the Environment Created by Multiple Microvibration Sources," Journal of Sound and Vibration, Vol. 344, 2015, pp. 138-157. doi: 10.1016/j.jsv.2015.01.035

[4] Remedia, M., Aglietti, G.S., Richardson, G., and Sweeting, M., "Integrated Semiempirical Methodology for Microvibration Prediction," AIAA Journal, Vol. 53, No. 5, 2015, pp. 1236-1250. doi: 10.2514/1.J053339

[5] Desmet, W., Pluymers, B, and Atak, O., MID-FREQUENCY - CAE Methodologies for Mid-frequency Analysis in Vibrations and Acoustics, KU Leuven, 2012

[6] ECSS, ECSS-E-HB-32-26A Spacecraft Mechanical Loads Analysis Handbook , ESA Requirements and Standards Division, 2013 
[7] Bialke, B., "Microvibration Disturbance Sources in Reaction Wheels and Momentum Wheels," European Conference on Spacecraft Structures, Materials \& Mechanical Testing , Noordwijk, The Netherlands, 1996

[8] Meza, L., Tung, F., Anandakrishnan, S., Spector, V., and Hyde, T. T., "Line of Sight Stabilization of James Webb Space Telescope," 27th Annual AAS Guidance and Control Conference , Breckenridge, $\mathrm{CO}, 2005$

[9] Zhang, Z., Yang, L., and Pang, S., "Jitter Environment Analysis for Micro-precision Spacecraft," Spacecraft Enviroment Engineering, Vol. 26, No. 6, 2009, pp. 528-534

[10] Miller, S. E., Kirchman, P., and Sudey, J., "Reaction Wheel Operational Impacts on the GOES-N Jitter Environment," AIAA Guidance, Navigation and Control Conference and Exhibit , South Carolina, USA, 2007

[11] Bialke, B., "Microvibration Disturbance Fundamentals for Rotating Mechanisms," 34th Annual Guidance and Control Conference, Breckenridge, CO, 2011

[12] Elias, L. M., Dekens, F. G., Basdogan, I., Sievers, L. A., and Neville, T., "A Methodology for Modeling the Mechanical Interaction between a Reaction Wheel and a Flexible Structure," Interferometry in Space, Waikoloa, HI, USA, 2003

[13] Takahara, O., Ichimoto, K., Kosugi, T., Shimada, S., Yoshida, N., "Evaluation of Pointing Error for SOLAR-B Using Optical Measurement," Uchu Kagaku Gijutsu Rengo Koenkai Koenshu , Vol. 50, 2006, pp. $271-275$

[14] Masterson, R. A., Miller, D. W., and Grogan, R. L., "Development and Validation of Reaction Wheel Disturbance Models: Empirical Model," Journal of Sound and Vibration, Vol. 249, No. 3, 2002, pp. $575-598$

[15] Elias, L. M., and Miller, D. W., "A Coupled Disturbance Analysis Method using Dynamic Mass Measurement Techniques," 43rd AIAA/ASME/ASCE/AHS/ASC Structures, Structural Dynamics, and Materials Conference, Denver, Colorado, 2002

[16] Aglietti, G. S., Langley, R. S., Rogers, E., and Gabriel, S. B., "Model Building and Verification for Active Control of Microvibrations with Probabilistic Assessment of the Effects of Uncertainties," Proceedings of the Institution of Mechanical Engineers, Part C: Journal of Mechanical Engineering Science , Vol. 218, No. 4, 2004, pp. 389-399

[17] Zhang, Z., Aglietti, G. S., and Zhou, W., "Microvibrations Induced by a Cantilevered Wheel Assembly with a Soft-Suspension System," AIAA Journal, Vol. 49, No. 5, 2011, pp. 1067-1079 
[18] Zhou, W., Aglietti, G. S., and Zhang, Z., "Modelling and Testing of a Soft Suspension Design for a Reaction/momentum Wheel Assembly," Journal of Sound and Vibration, Vol. 330, No. 18, 2011, pp. $4596-4610$

[19] Zhang, Z., Aglietti, G. S., Ren, W., and Addari, D., "Microvibration Analysis of a Cantilever Configured Reaction Wheel Assembly," Advances in Aircraft and Spacecraft Science, An Int'l Journal , Vol. 1, No. 4, 2014, pp. 379-398. doi: 10.12989/aas.2014.1.4.379

[20] Aglietti, G. S., Gabriel, S., Langley, R. S., and Rogers, E., "A Modeling Technique for Active Control Design Studies with Application to Spacecraft Microvibrations,", The Journal of the Acoustical Society of America, Vol. 102, No. 4, 1997, pp. 2158-2166

[21] Wang, P., and Zhang, X., "Damper Based on Electromagnetic Shunt Damping Method," International Journal of Applied Electromagnetics and Mechanics , Vol. 33, No. 3, 2010, pp. 1425-1430

[22] Richardson, G., Smet, G., and Aglietti, G. S., "Managing Micro-Vibration on the SSTL300-S1 a 400 Kg 1m Resolution Earth Imaging Spacecraft," Proceeding of European Conference on Spacecraft Structures, Materials 86 Environmental Testing, Braunschweig, Germany, 2014

[23] Yoshida, N., "Pointing Accuracy, Vibration and Control of Artificial Satellite," Nippon Kikai Gakkai Kansai Shibu Teiji Sokai Koenkai Koen Ronbunshu , Vol. 81, 2006, pp. 35-38

[24] Shimizu, T., Nagata, S., Tsuneta, S., Tarbell, T., Edwards, C., Shine, R., Hoffmann, C., Thomas, E., Sour, S. and Rehse, R., "Image Stabilization System for Hinode (Solar-B) Solar Optical Telescope," Solar Physics, Vol. 249, No. 2, 2008, pp. 221-232. doi: 10.1007/s11207-007-9053-z

[25] Laurens, P., Decoux, E., and Janvier, M., "SOHO Microvibrations: Analyses, Tests and Flight Results," Spacecraft Guidance, Navigation and Control Systems, Proceedings of the 3rd ESA International Conference, Noordwijk, the Netherlands, 1997

[26] Taniwaki, S., and Ohkami, Y., "Experimental and Numerical Analysis of Reaction Wheel Disturbances," JSME International Journal Series C Mechanical Systems, Machine Elements and Manufacturing , Vol. 46, No. 2, 2003, pp. 519-526

[27] Taniwaki, S., Kudo, M., Sato, M., and Ohkami, Y., "Analysis of Retainer Induced Disturbances of Reaction Wheel," Journal of System Design and Dynamics , Vol. 1, No. 2, 2007, pp. 307-317

[28] Masterson, R. A., Miller, D. W., and Grogan, R. L., Development and Validation of Empirical and Analytical Reaction Wheel Disturbance Models , Ph.D. Thesis, Massachusetts Institute of Technology, 1999

[29] Elias, L. M., and Miller, D. W., Dynamics of Multi-Body Space Interferometers Including Reaction Wheel Gyroscopic Stiffening Effects: Structurally Connected and Electromagnetic Formation Flying 
Architectures, Ph.D. Thesis, Massachusetts Institute of Technology, 2004

[30] Zhao, Y., Zhang, P. F., and Cheng, W., "Measurement and Study of Disturbance Characteristics of Reaction Wheel Assembly," Journal of Experimental Mechanics, Vol. 24, No. 6, 2009, pp. 532-538

[31] Zhang, Z., Ren, W., and Aglietti, G. S., "Coupled Disturbance Modelling and Validation of a Reaction Wheel Model," European Conference on Spacecraft Structures, Materials $\&$ Environmental Testing , Noordwijk, The Netherlands, 2012

[32] Basdogan, I., Elias, L. M., Dekens, F., and Sievers, L., "Predicting the Optical Performance of the Space Interferometry Mission using a Modeling, Testing, and Validation," Journal of Vibration and Acoustics , Vol. 129, No. 2, 2007, pp. 148-157

[33] Zhang, Z., Aglietti, G. S., and Ren, W., "Coupled Microvibration Analysis of a Reaction Wheel Assembly Including Gyroscopic Effects in its Accelerance," Journal of Sound and Vibration, Vol. 332, No. 22, 2013, pp. 5748-5765

[34] Addari, D., Aglietti, G. S., Remedia, M., Richardson, G., and LePage, B., "Inspecting the Characterisation of Microvibration Sources," 65th International Astronautical Congress, Toronto, Canada, 2014

[35] Addari, D., Aglietti, G. S., and Remedia, M., "Investigating Microvibration Sources Modelling,", 13th European Conference on Spacecraft Structures, Materials \& Environmental Testing , Braunschweig, Germany, 2014

[36] Hazewinkel, M., Encyclopedia of Mathematics: an Updated and Annotated Translation of the Soviet Mathematical Encyclopedia, Springer Netherlands, 1997

[37] Laurens, P., and Decoux, E., "Microdynamic Behaviour of Momentum and Reaction Wheels," Proceedings of the Second Space Microdynamics and Accurate Symposium , Toulouse, France, 1997

[38] Liu, K. C., Maghami, P., and Blaurock, C., "Reaction Wheel Disturbance Modeling, Jitter Analysis, and Validation Tests for Solar Dynamics Observatory," AIAA Guidance, Navigation and Control Conference and Exhibit, Honolulu, Hawaii, 2008 